\title{
DISTANCE TRANSFORMATION FOR NETWORK DESIGN PROBLEMS
}

\author{
A. RIDHA MAHJOUB *, MICHAEL POSS ${ }^{\dagger}$, LUIDI SIMONETTI ${ }^{\ddagger}$, AND EDUARDO \\ UCHOA $\S$
}

\begin{abstract}
We propose a new generic way to construct extended formulations for a large class of network design problems with given connectivity requirements. The approach is based on a graph transformation that maps any graph into a layered graph according to a given distance function. The original connectivity requirements are in turn transformed into equivalent connectivity requirements in the layered graph. The mapping is extended to the graphs induced by fractional vectors through an extended linear integer programming formulation. While graphs induced by binary vectors are mapped to isomorphic layered graphs, those induced by fractional vectors are mapped to a set of graphs having worse connectivity properties. Hence, the connectivity requirements in the layered graph may cut off fractional vectors that were feasible for the problem formulated in the original graph. Experiments over instances of the Steiner Forest and Hop-constrained Survivable Network Design problems show that significant gap reductions over the state-of-the art formulations can be obtained.
\end{abstract}

Key words. Extended Formulations, Network Design, Benders decomposition.

AMS subject classifications.

1. Introduction. Let $G=(V, E)$ be an undirected graph with $n$ vertices and $m$ edges with positive $\operatorname{costs} c_{e}, e \in E$. Let $D \subseteq V \times V$ be a set of demands, each demand $(u, v) \in D$ has its connectivity requirements: the existence of a certain number of $(u, v)$-paths, possibly those paths may need to satisfy some side constraints. The class of Network Design Problems (NDPs) considered in this paper consists in finding a subgraph of $G$ with minimum cost satisfying the connectivity requirements of all demands. For examples:

- Steiner Tree Problem (STP): the input gives a set $T \subseteq V$ of terminals. $D$ can be defined as $\{(u, v): u, v \in T, u \neq v\}$. The connectivity requirement of a demand $(u, v) \in D$ is the existence of a path joining $u$ and $v$.

- Steiner Forest Problem (SFP): An arbitrary set $D$ is given as input. The connectivity requirement is the existence of a path joining the vertices in each demand.

- Hop-constrained Steiner Tree Problem (HSTP): the input gives a set $T \subseteq V$ of terminals, a root vertex $r \in T$ and an integer $H$. The demand-set $D$ is defined as $\{(r, v): v \in T \backslash\{r\}\}$. The connectivity requirement is the existence of a path with at most $H$ edges (hops) joining the vertices in each demand.

- Hop-constrained Survivable Network Design Problem (HSNDP): the set $D$ and integers $H$ and $K$ are given as input. The connectivity requirement of a demand $(u, v) \in D$ is the existence of $K$ edge-disjoint $(u, v)$-paths, each path having at most $H$ edges.

Several other NDPs found in the literature also belong to this class, the connectivity

\footnotetext{
*Université Paris-Dauphine, Place du Maréchal De Lattre de Tassigny, 75775, Paris Cedex 16, France (mahjoub@lamsade.dauphine.fr).

†UMR CNRS 5506 LIRMM, Université de Montpellier 2, 161 rue Ada, 34392 Montpellier Cedex 5, France. (michael.poss@lirmm.fr).

${ }^{\ddagger}$ COPPE - PESC, Federal University of Rio de Janeiro, Cidade Universitria, Centro de Tecnologia, Bloco H, zip 21941-972, Rio de Janeiro, Brazil. (luidi@ic.uff.br).

$\S$ Universidade Federal Fluminense, Rua Passo da Pátria, 156 CEP 24210-240, Niterói, Brazil (uchoa@producao.uff.br).
} 
requirement may ask for a number of node-disjoint paths, side constraints may include maximum delay, etc.

On all those cases, the natural formulations over the design variables $x_{e}, e \in E$, take the following format:

$$
\begin{array}{cl}
\operatorname{Min} & \sum_{e \in E} c_{e} x_{e} \\
\text { S.t. } & x \in P(u, v) \forall(u, v) \in D \\
& x_{e} \text { binary } \forall e \in E,
\end{array}
$$

where the binary points in polyhedra $P(u, v)$ correspond to all subgraphs satisfying the connectivity requirements of demand $(u, v)$. Even on cases when each polyhedron $P(u, v)$ is integral, i.e., when the formulation already contains the best possible inequalities that are valid for each individual demand, the overall formulation is often still weak.

For example, consider the classic STP, where the natural Formulation (1) corresponds to the one proposed by Aneja [1]. In that case, constraints (1b) are given by the so called undirected cut constraints: for each cut separating two terminals $u$ and $v$, there must be at least an edge in the solution. The linear relaxation of Aneja's formulation does not provide very good bounds. Even when the formulation is reinforced with sophisticated cuts discovered after polyhedral investigation $[4,5]$, the duality gaps may be still significant. Fortunately, Wong [23] proposed a much better STP formulation. The main observation is that the set of demands $D=\{(u, v): u, v \in T, u \neq v\}$ can be replaced by $D^{\prime}=\{(r, v): v \in T \backslash\{r\}\}$, where $r \in T$ is an arbitrarily chosen terminal, without changing the optimal solution. Then, $G$ can be replaced by a bidirected graph $G^{\prime}=(V, A)$ where $A$ has two opposite arcs $(i, j)$ and $(j, i)$ for each edge $e=\{i, j\}$ in $E$, with $c((i, j))=c((j, i))=c(e)$. The problem now consists in looking for a minimum cost subgraph of $G^{\prime}$ having a directed path from $r$ to every other terminal. The proposed formulation uses directed cut constraints: for each directed cut separating $r$ from a terminal, there must be at least an arc in the solution. Wong's formulation is remarkably strong. Its duality gaps are less than $0.1 \%$ on almost all SteinLIB instances [14], except on the artificial instances created with the intent of being hard. In fact, some of the most effective STP codes of today, which are capable of solving non-artificial instances (like those from VLSI design) with tenths of thousands of vertices, usually do not bother to separate cuts other than the simple directed cut constraints. Instead, their algorithmic effort is focused on devising graph reductions and dual ascent procedures in order to speedup the solution of that linear relaxation $[19,20]$.

Of course, one would like to have similar reformulation schemes able to produce strong bounds for other NDPs. An advance in that direction was the work by Gouveia et al. [11] on hop/diameter constrained spanning/Steiner Tree Problems. On all those cases it was possible to show that the problem could be transformed into a STP over a directed graph composed of up to $H$ layers, each layer consisting of copies of the original graph. Additional arcs joining the layers and a few extra vertices/arcs are required. By applying Wong's directed cut formulation over the transformed graphs, very small gaps are obtained. Even though the layered graph approach multiplies the size of the graph, it still allowed the solution of instances with hundreds of vertices. Recently, the layered graph approach was successfully applied on a number of other NDPs $[21,16,9,10]$, effective techniques were introduced the cope with the large transformed graphs and to handle other kinds of constraints. 


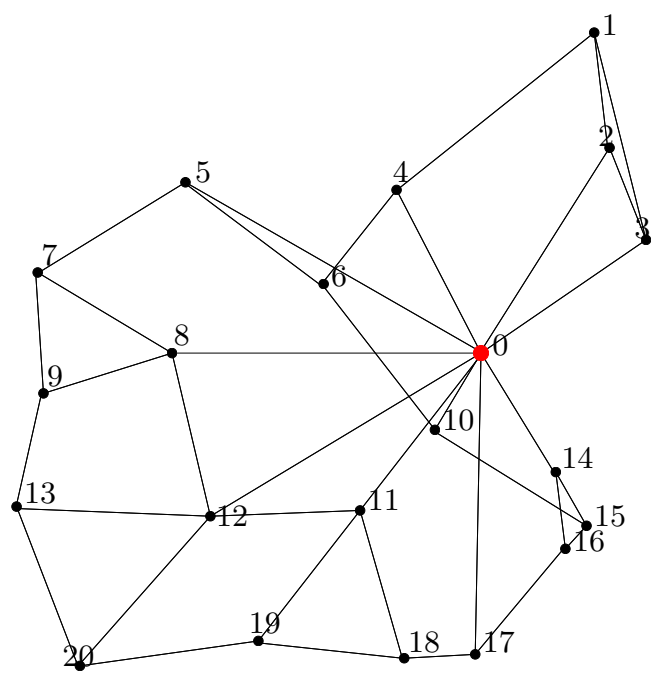

FIG. 1. Optimal solution of a HSNDP instance with $K=3$ and $H=3 ; n=21, D=\{(0, v)$ : $v=1, \ldots, 20\}$, complete graph, Euclidean costs.

Nevertheless, we should remark that all those strong reformulation schemes are restricted to relatively simple NDPs. As the reformulations depend on transforming the problem into a directed $S T P$, they are restricted to problems where the optimal solution topology should be a single tree. NDPs with more complex topologies do not seem to admit directed formulations.

- Sometimes this is related to the fact that both orientations of an edge can be used in the paths connecting different demands. Figure 1 shows the optimal solution of a HSNDP instance with demand-set $D=\{(0, v): v=1, \ldots, 20\}$, $K=3$ and $H=3$. Even tough all demands include vertex 0 , which makes this vertex a natural root for the problem, it can be seen that the same edge can be used in different directions by different demands. For example, demand $(0,7)$ is connected by paths $0-5-7,0-8-9-7$, and $0-12-8-7$; demand $(0,5)$ is connected by paths $0-5,0-10-6-5$ and $0-8-7-5$. Suppose that $G$ is transformed into a bidirected graph. The corresponding directed solution would need to pay for both $\operatorname{arcs}(5,7)$ and $(7,5)$, which is not correct.

- Even the seemingly simple SFP, where the solution may be formed by a set of disconnected subtrees, does not seem to admit a strong reformulation by turning it into a directed problem over a transformed graph. Here, the difficulty lies in the fact that it is not possible to know beforehand which demands will be connected by the same subtree. In fact, the strongest known reformulation for the SPF, proposed in [15], is only slightly better than the natural Formulation (1).

The Distance Transformation (DT) is an original reformulation technique proposed to obtain stronger formulations for general NDPs, including those where the currently know reformulation techniques do not seem to work. Starting from Formu- 
lation (1), the resulting reformulation will have the following format:

$$
\begin{gathered}
\operatorname{Min} \quad \sum_{e \in E} c_{e} x_{e} \\
\text { S.t. } A x+B w+C y \geq b \\
(w, y) \in P^{\prime}(u, v) \quad \forall(u, v) \in D \\
x_{e} \text { binary } \quad \forall e \in E,
\end{gathered}
$$

where $A, B, C$ and $b$ are matrices of appropriated dimensions. Constraints (2b) are problem-independent. They are devised to transform a solution over the variables $x$ into a distance expanded solution over the new variables $w$ and $y$. The original connectivity constraints in each $P(u, v)$, over the $x$ variables, are transformed into new connectivity constraints $P^{\prime}(u, v)$, over the $(w, y)$ variables.

The purpose of DT can be informally described as follows. Let $x$ be a binary vector in $\{0,1\}^{m}$ and $G(x)=(V, E(x))$ be the subgraph induced by $x$. The distance transformation maps $G(x)$ into a subgraph of the layered graph that consists of $n+1$ copies of $G$ plus edges between adjacent layers. The mapping considers a chosen source subset $S \subseteq V$ and sends each vertex $i \in V$ to a vertex in layer $l, 0 \leq l \leq n$, according to its distance from set $S$. Each edge $\{i, j\} \in G(x)$ is sent to an edge in the layered graph according to the layers of $i$ and $j$. The transformed graph is represented by variables $w$ and $y$, the mapping is encoded in Constraints (2b). The interest of the transformation is the following. If $x$ is integral, the transformed graph is isomorphic to $G(x)$. However, its extension to graphs $G(x)$ induced by fractional vectors leads to transformed graphs that are less connected than $G(x)$. Hence, the corresponding $(w, y)$ fractional solution is much more likely to be cut by inequalities (2c).

At first glance, this concept may seem similar to the layered formulations used in $[11,21,16,9,10]$. They are, however, completely different. In those previous works, the role of the layered graphs was to model connectivity requirements with hop/distance/delay constraints. In contrast, the DT is not linked to any particular kind of connectivity requirements. In fact, those techniques are orthogonal: it is possible to combine DT and layered formulations.

1.1. Contributions and structure of the paper. The idea behind DT has its origin in an extended formulation (denoted as DT-HOP MCF) introduced in Mahjoub et al. [18] for the HSNDP. The numerical results obtained were very positive, significant gap reductions with respect to previous known formulations led to the solution of several open instances from the literature. There was however no theoretical justification for the gap reductions obtained by what seemed to be an ad-hoc reformulation for that specific network design problem.

The purpose of this paper is to introduce the Distance Transformation, a generic graph transformation that works for the aforementioned class of network design problems. We show in Section 2.1 how the distance transformation is naturally defined as a graph transformation. The transformation is extended to graphs defined by fractional vectors by using convexity arguments. We explain then in Section 2.2 how this ideal distance transformation reduces the connectivity of fractional vectors by splitting nodes. Section 3 studies linear programming formulations for the distance transformation. A natural formulation is presented in Section 3.1. Section 3.2 studies relaxations of the formulation to make it more effective computationally. Section 4 shows how basic connectivity requirements can be incorporated into the linear programming formulations through inequalities or flow formulations. The resulting formulations are 


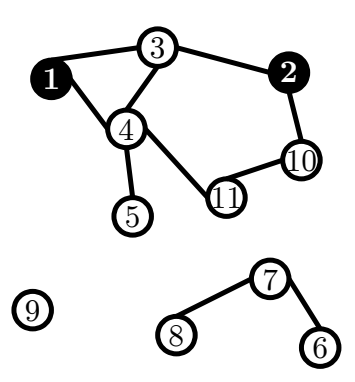

(a) $G$

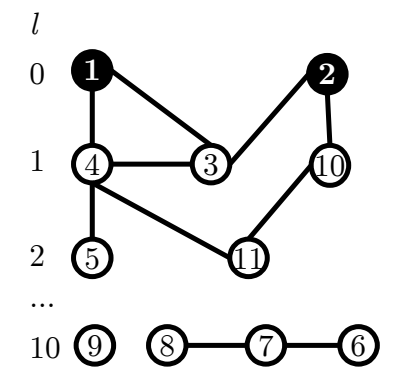

(b) $D T_{S}(G)$

Fig. 2. Original graph $G$ and $D T_{S}(G)$ with $S=\{1,2\}$.

illustrated numerically in Section 5 on two network design problems: the Steiner Forest Problem and the Hop-constrained Survivable Network Design Problem. The paper is finally concluded in Section 6.

1.2. Notations. Let $F(0,1)=[0,1]^{m} \backslash\{0,1\}^{m}$ be the set of vectors in the unit hypercube having at least one fractional component. Undirected edges are denoted by $\{i, j\}$, and directed edges are denoted by $(i, j)$. In addition, we use the following conventions in all figures in the paper. The set of source nodes $S$ contains the vertices filled in black. Plain edges and nodes have weights equal to 1 , while dotted edges and nodes with dotted circle have weights equal to 0.5 . The set of extreme points of a polytope $P$ is denoted as $\operatorname{ext}(P)$.

2. The unitary distance transformation. We introduce formally the distance transformation in Subsection 2.1 and study in Subsection 2.2 the connectivity of the layered graphs obtained for fractional vectors $x$. Linear programming formulations for DT shall be discussed in the next section.

2.1. Definition. The distance transformation considers an arbitrary subset of root nodes $S \subset V$ and maps any subgraph $G^{\prime}=\left(V, E^{\prime}\right)$ of $G=(V, E)$ to a layered graph. The mapping is based on the distance in $G^{\prime}$ between any node $i \in V$ and $S$. Define the layered and undirected graph $\mathbf{G}=(\mathbf{V}, \mathbf{E})$, where $\mathbf{V}=V^{0} \cup \ldots \cup V^{n}$, with $V^{l}=\left\{i^{l}: i \in V\right\}$ for each $l=0, \ldots, n$ and $i^{l}$ be the copy of vertex $i$ in the $l$-th level of graph $\mathbf{G}$. Then, the edge set is defined by $\mathbf{E}=\left\{\left\{i^{l_{1}}, j^{l_{2}}\right\} \mid\{i, j\} \in E, 0 \leq l_{1} \leq\right.$ $\left.n-2, l_{1}-1 \leq l_{2} \leq l_{1}+1\right\} \cup\left\{\left\{i^{n}, j^{n}\right\} \mid\{i, j\} \in E\right\}$. Let $\mathcal{P}(G)$ be the set of all subgraphs of $G$ that contain all nodes of $V$ and $\mathcal{P}(\mathbf{G})$ be the set of all subgraphs of $\mathbf{G}$ that contain exactly one copy of each node of $V$, formally: $\mathcal{P}(G)=\left\{G^{\prime}=\left(V, E^{\prime}\right) \mid E^{\prime} \subseteq E\right\}$ and $\mathcal{P}(\mathbf{G})=\left\{\mathbf{G}^{\prime}=\left(\mathbf{V}^{\prime}, \mathbf{E}^{\prime}\right) \mid \mathbf{V}^{\prime} \subseteq \mathbf{V}\right.$ and $\left.\left|\mathbf{V} \cap\left\{i^{l}: 0 \leq l \leq n\right\}\right|=1, \forall i \in V, \mathbf{E}^{\prime} \subseteq \mathbf{E}\right\}$.

The distance transformation is a function $D T_{S}: \mathcal{P}(G) \rightarrow \mathcal{P}(\mathbf{G})$ that maps any subgraph $G^{\prime} \in \mathcal{P}(G)$ to layered graph $D T_{S}\left(G^{\prime}\right) \in \mathcal{P}(\mathbf{G})$ defined as follows. Let $\operatorname{dist}_{G^{\prime}}(i, S)$ be the length of the shortest path in $G^{\prime}$ connecting $i$ to a node in $S$ or $\infty$ if no such path exists. For each $i \in V$ such that $\operatorname{dist}_{G^{\prime}}(i, S)<\infty$, vertex $i^{\operatorname{dist}_{G^{\prime}}(i, S)}$ belongs to $D T_{S}\left(G^{\prime}\right)$. Otherwise, $i^{n} \in D T_{S}\left(G^{\prime}\right)$. For any $\{i, j\} \in G^{\prime},\left\{i^{l_{1}}, i^{l_{2}}\right\}$ belongs to $D T_{S}\left(G^{\prime}\right)$ if and only if $l_{1}=\operatorname{dist}_{G^{\prime}}(i, S)$ and $l_{2}=\operatorname{dist}_{G^{\prime}}(j, S)$. Remark that for any $\{i, j\} \in G^{\prime},\left|\operatorname{dist}_{G^{\prime}}(i, S)-\operatorname{dist}_{G^{\prime}}(j, S)\right| \leq 1$. In other words, the images of adjacent vertices in $G^{\prime}$ lie in adjacent levels in $D T_{S}\left(G^{\prime}\right)$. Figure 2 shows an example of distance transformation. A crucial property of the distance transformation is that $G^{\prime}$ and $D T_{S}\left(G^{\prime}\right)$ are isomorphic. 
Any graph in $\mathcal{P}(G)$ can be characterized as $G(x)=(V, E(x))$ where $x \in\{0,1\}^{m}$ is a binary vector whose component $\{i, j\}$ is equal to 1 if and only if $\{i, j\} \in E(x)$; we say that $G(x)$ is induced by $x$. We can also describe a graph $\mathbf{G}^{\prime}=\left(\mathbf{V}^{\prime}, \mathbf{E}^{\prime}\right)$ in $\mathcal{P}(\mathbf{G})$ using vectors $w \in\{0,1\}^{n(n+1)}$ and $y \in\{0,1\}^{(3 n-1) m}$, defined as follows:

- $w_{i}^{l}=1$ iff $i^{l} \in \mathbf{V}^{\prime}$

- $y_{i j}^{l_{1} l_{2}}=1$ iff $\left\{i^{l_{1}}, j^{l_{2}}\right\} \in \mathbf{E}^{\prime}$.

Conversely, given binary vectors $w$ and $y, \mathbf{G}(w, y)$ denotes the subgraph of $\mathbf{G}$ that contains the vertices (resp. edges) associated to the components of $w$ (resp. $y$ ) equal to 1 . Hence, we also define the function $D T_{S}:\{0,1\}^{m} \rightarrow\{0,1\}^{n(n+1)+(3 n-1) m}$ as $D T_{S}(x)=(w, y)$ where $(w, y)$ characterizes the graph $D T_{S}(G(x))$.

In this paper, we address network design problems formulated as linear programs with binary variables. In this context, $x$ is a vector of optimization variables comprised between 0 and 1 . To use the distance transformation, we must describe $D T_{S}$ through a system of linear constraints such that for each $x \in\{0,1\}^{m},(x, w, y)$ is feasible if and only if $(w, y)=D T_{S}(x)$. Certainly, the smallest polytope defined by such constraints is

$$
P_{S}=\operatorname{conv}\left\{\left(x, D T_{S}(x)\right), x \in\{0,1\}^{m}\right\} .
$$

Using $P_{S}$, we are able to define $D T_{S}$ that is defined for any vector in $x \in[0,1]^{m}$, fractional or not, as the following projection:

$$
D T_{S}(x)=\left\{(w, y) \mid(x, w, y) \in P_{S}\right\} .
$$

The more general definition (4) is compatible with the previous definition for integral vectors because whenever $x$ is integral $D T_{S}(x)$ reduces to the singleton $\left\{D T_{S}(x)\right\}$. Otherwise, set $D T_{S}(x)$ is a polytope with non-zero dimension. Therefore, $D T_{S}$ : $[0,1]^{m} \rightrightarrows[0,1]^{n(n+1)+(3 n-1) m}$ is a point-to-set mapping. We introduce next a straightforward characterization of $D T_{S}(x)$.

TheOrem 1. Let $x^{q}, q=1, \ldots, 2^{m}$, be the enumerated set of all vectors in $\{0,1\}^{m}$. A vector $(w, y)$ belongs to $D T_{S}(x)$ if and only if there exists a vector of convex multipliers $\lambda$ such that $x=\sum_{q=1}^{2^{m}} \lambda^{q} x^{q}$ and $(w, y)=\sum_{q=1}^{2^{m}} \lambda^{q} D T_{S}\left(x^{q}\right)$.

Proof. Direct from (3) and (4).

Extending the previous definitions to fractional vectors, we can define $G(x)$ and $\mathbf{G}(w, y)$ as the graphs induced by the positive components of $x$ and $(w, y)$, respectively. We provide next an example of $D T_{S}\left(x^{\prime}\right)$ for the fractional vector $x^{\prime}=(0.5,0.5,0.5)$ associated to the graph $G^{\prime}=\left(V^{\prime}, E^{\prime}\right)$ with $V^{\prime}=\{1,2,3\}$ and $E^{\prime}=\{\{1,2\},\{1,3\},\{2,3\}\}$, and $S=\{1\}$. Figures $3(\mathrm{a})-3(\mathrm{~h})$ represent the graphs induced by the vectors of $D T_{S}(x)$ for each $x \in\{0,1\}^{3}$. In view of Theorem 1 , any element in $D T_{S}\left(x^{\prime}\right)$ is obtained from expressing $x^{\prime}$ as a specific convex combination of the vectors in $\{0,1\}^{3}$. Hence, one readily verifies by examination that $\operatorname{ext}\left(D T_{S}\left(x^{\prime}\right)\right)$ contains the vectors that induce the four graphs from Figure 4, which correspond to four different convex combinations describing $x^{\prime}$. One can see the splitting of nodes occurring in the graphs induced by all vectors of $\operatorname{ext}\left(D T_{S}\left(x^{\prime}\right)\right)$. The splitting of nodes corresponds to fractional values of $w$. As will be seen in the next section, the splitting of nodes for all vectors of $\operatorname{ext}\left(D T_{S}\left(x^{\prime}\right)\right)$ is a necessary condition for DT to be useful.

This does not always happens. Consider a similar example, except that $S=\{1,2\}$. The graphs for vectors in $\operatorname{ext}\left(D T_{S}\left(x^{\prime}\right)\right)$ are shown in Figure 5 . We see that the left graph does not contain split nodes. Hence, that choice of $S$ would not give a useful transformation. 


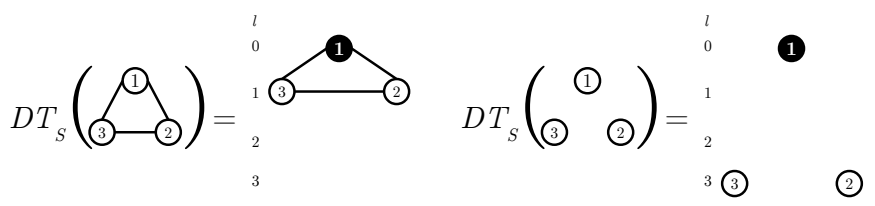

(a) $G^{\prime}\left(x^{1}\right)$

(b) $G^{\prime}\left(x^{2}\right)$

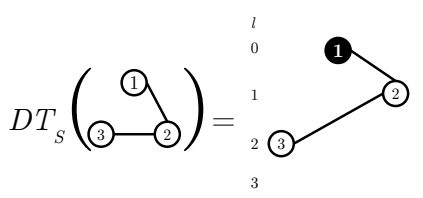

(c) $G^{\prime}\left(x^{3}\right)$

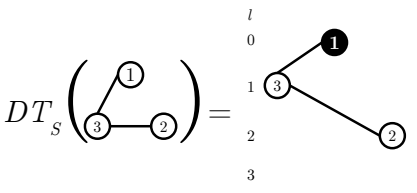

(d) $G^{\prime}\left(x^{4}\right)$

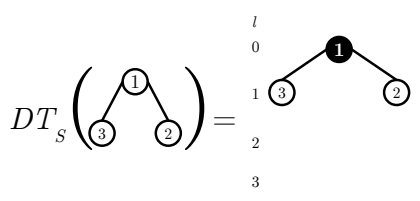

(e) $G^{\prime}\left(x^{5}\right)$

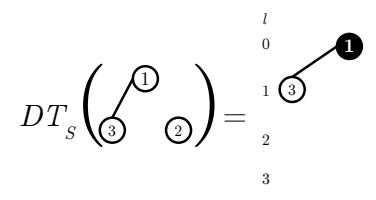

(g) $G^{\prime}\left(x^{7}\right)$

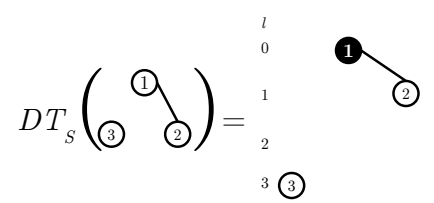

(f) $G^{\prime}\left(x^{6}\right)$

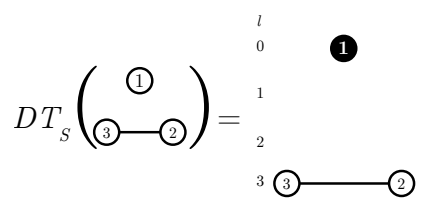

(h) $G^{\prime}\left(x^{8}\right)$

FIG. 3. $D T_{S}(x)$ for each $x \in\{0,1\}^{3}$.

2.2. Connectivity of $D T_{S}$. The distance transformation translates the original network design problem defined in $G$ into a network design problem defined in $\mathbf{G}$, by importing to $\mathbf{G}$ the connectivity requirements described in $G$. In this section, we illustrate how this is done for the simpler case of demands requiring the existence of $K$ edge-disjoint paths between some pairs of vertices. To this end, we add up to $2|D|$ supervertices to $\mathbf{G}$ and $\mathbf{G}(w, y)$, obtaining $\overline{\mathbf{G}}=(\overline{\mathbf{V}}, \overline{\mathbf{E}})$ and $\overline{\mathbf{G}}(w, y)=(\overline{\mathbf{V}}(w), \overline{\mathbf{E}}(w, y))$, respectively. Namely, for each demand $(u, v) \in D$, we create two supervertices $s(u)$ and $t(v)$ respectively linked to vertices $u^{l}$ and $v^{l}$ by directed edges $\left(s(u), u^{l}\right)$ and $\left(v^{l}, t(v)\right)$ for each $0 \leq l \leq n$. For any $x \in\{0,1\}^{m}$, the requirement of the existence in $G(x)$ of $K$ edge-disjoint $(u, v)$-paths becomes the requirement of the existence in $\overline{\mathbf{G}}(w, y)$ of $K$ edge-disjoint $(s(u), t(v))$-paths.

To model these connectivity requirements by linear constraints, we need to consider graphs $G(x)$ and $\overline{\mathbf{G}}(w, y)$ as weighted graphs. The weight on any edge in $G(x)$ is equal to the value of the associated component of $x$. For $\overline{\mathbf{G}}(w, y)$, we must distinguish between the undirected edges, and the directed ones that link $\mathbf{G}(w, y)$ to the supervertices. The weight on any undirected edge is equal to the value of the associated component of $y$, while the weight on any directed edge linking $s(u)$ (resp. $t(v))$ and $u^{l}$ (resp. $v^{l}$ ) is equal to $K w_{u}^{l}$ (resp. $K w_{v}^{l}$ ). We define next the connectivity of weighted graph $G(x)$ as the vector $C(x) \in \mathbb{R}_{+}^{|D|}$ with $C_{u v}(x)$ equal to the maximum flow between $u$ and $v$ in $G(x)$. Similarly, we define the connectivity of weighted 


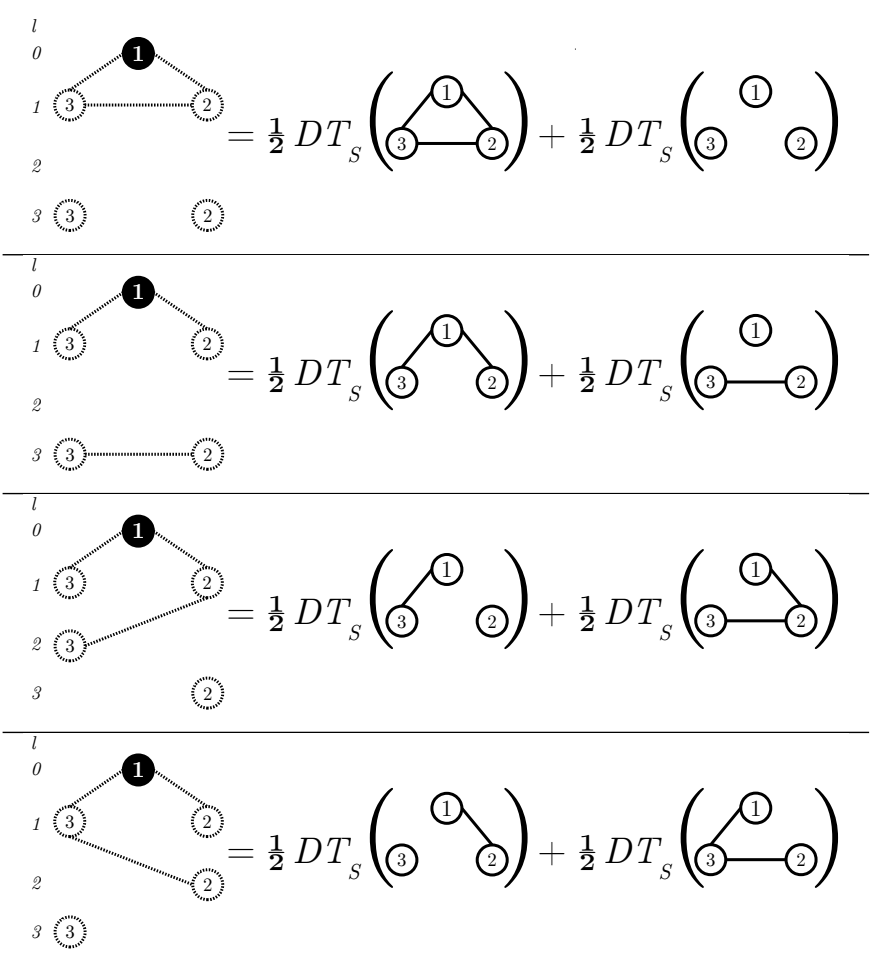

FIG. 4. Graphs in $\operatorname{ext}\left(D T_{S}\left(G^{\prime}(x)\right)\right)$ when $S=\{1\}$.

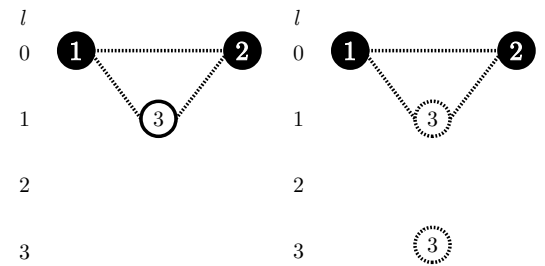

FIG. 5. Graphs in $\operatorname{ext}\left(D T_{S}\left(G^{\prime}(x)\right)\right)$ when $S=\{1,2\}$.

graph $\overline{\mathbf{G}}(w, y)$ as the vector $C(w, y) \in \mathbb{R}_{+}^{|D|}$ with $C_{u v}(w, y)$ equal to the maximum flow between the supervertices $s(u)$ and $t(v)$ in $\overline{\mathbf{G}}(w, y)$. With these definitions, the network design problem defined in $G$ with optimization variables $x$ and connectivity requirements

$$
C_{u v}(x) \geq K, \quad \forall(u, v) \in D,
$$

can be reformulated as a network design problem defined in $\overline{\mathbf{G}}$ with optimization variables $x, w$, and $y$, and containing two groups of constraints:

1. Constraints specifying that $(x, w, y) \in P_{S}$;

2. Connectivity requirements constraints

$$
C_{u v}(w, y) \geq K, \quad \forall(u, v) \in D
$$

In the following we denote the feasibility set of constraints (5) by

$$
\mathcal{C}^{0}=\left\{x \in[0,1]^{m} \mid C_{u v}(x) \geq K, \forall(u, v) \in D\right\}
$$


and the projected feasibility set of constraints (6) by

$$
\mathcal{C}^{P_{S}}=\operatorname{Proj}_{x}\left\{(x, y, w) \in P_{S} \mid C_{u v}(w, y) \geq K, \forall(u, v) \in D\right\},
$$

where $\operatorname{Proj}_{x}(\mathcal{X})$ denotes the projection of set $\mathcal{X}$ on variables $x$. We also denote the convex hull of the feasible solutions of the network design problem defined by connectivity requirements (5) as

$$
\mathcal{C}^{o p t}=\operatorname{conv}\left\{x \in\{0,1\}^{m} \mid C_{u v}(x) \geq K, \forall(u, v) \in D\right\} .
$$

The result below shows that the approximation of $\mathcal{C}^{o p t}$ provided by $\mathcal{C}^{P_{S}}$ is tighter than the one provided by $\mathcal{C}^{0}$.

Proposition 2. For any connectivity requirements constraints of the form (5) and any $S$, it always holds that

$$
\mathcal{C}^{o p t} \subseteq \mathcal{C}^{P_{S}} \subseteq \mathcal{C}^{0} .
$$

Proof. Let $x \in\{0,1\}^{m}$ and $D T_{S}(x)=(w, y)$. The first inclusion follows from the fact that $G(x)$ and $\mathbf{G}(w, y)$ are isomorphic, and thus, $C_{u v}(x)=C_{u v}(w, y)$ for each $(u, v) \in D$. To prove the second inclusion, we first prove that

$$
C_{u v}(x) \geq C_{u v}(w, y), \quad \forall(u, v) \in D .
$$

Let $(u, v) \in D$ and let $g$ be any vector defining a flow from $s(u)$ to $t(v)$ in $\overline{\mathbf{G}}(w, y)$. Then, we can flatten $\overline{\mathbf{G}}(w, y)$ to obtain a flow $f$ from $u$ to $v$. Namely, we define the flow $f$ on edge $\{i, j\} \in G(x)$ as the sums of the flows described by $g$ on all $\left\{i^{l_{1}}, j^{l_{2}}\right\} \in \mathbf{G}(w, y)$. The flows on the directed edges linking the supervertices $s(u)$ and $t(v)$ to $\mathbf{G}$ do not matter since we are only interested in a flow from $u$ to $v$. It is easy to see that the resulting flow $f$ satisfies the capacity constraints, the balance constraints and conveys the same amount of flow from $u$ to $v$ that $g$ conveys from $s(u)$ to $s(v)$. Therefore, if $x \in \mathcal{C}^{P_{S}}$, then we also have that $x \in \mathcal{C}^{0}$, proving the inclusion.

The power of distance transformation lies in its reduction of the connectivity of the graphs induced by $(w, y) \in D T_{S}(x)$ for fractional vectors $x \in(0,1)^{m}$. Even when a fractional solution $x$ is not cut by connectivity requirement constraints (5), meaning that $x \in \mathcal{C}^{0}$, it may well happen that all $(w, y) \in D T_{S}(x)$ are cut by connectivity requirement constraints (6), implying $x \notin \mathcal{C}^{P_{S}}$. The next result provides an example of $x \in \mathcal{C}^{0} \backslash \mathcal{C}^{P_{S}}$.

Proposition 3. Consider the network design problem defined on graph $G^{\prime}$ from Figure 3 under the connectivity requirements $C_{12}(x) \geq 1$ and $C_{13}(x) \geq 1$. It holds that

$$
\mathcal{C}^{P_{S}} \subset \mathcal{C}^{0}
$$

Proof. The inclusion follows from Proposition 2. To see that the inclusion is strict, we show that the fractional solution $x$ defined by $x_{12}=x_{23}=x_{13}=0.5$ belongs to $\mathcal{C}^{0} \backslash \mathcal{C}^{P_{S}}$. Clearly, $x \in \mathcal{C}^{0}$ since we can use the cycle to send half a unit in each direction for both demands in $D$. To see that $x \notin \mathcal{C}^{P_{S}}$, we must show that all $(w, y) \in D T_{S}(x)$ violate $C_{12}(w, y) \geq 1$ or $C_{13}(w, y) \geq 1$. First, we remark that

$$
\sum_{l=0}^{n} w_{i}^{l}=1, \quad \forall i \in V
$$


because any $(w, y) \in D T_{S}(x)$ can be extended to $(x, w, y) \in P_{S}$, which can be written as a convex combination of binary vectors $(x, w, y)$ satisfying equation (9). Consider then the four extreme points of $D T_{S}(x)$, which are depicted in Figure 4 where all edges have values equal to 0.5 . We can see that for each of these graphs, vertex 2 or vertex 3 (or both) belongs to the level 3 , so that the corresponding value of $w_{i}^{3}=0.5$ for $i=2$ or 3 . Since level 3 is not connected to 1, 0.5 units of flow cannot reach vertex 1 and equation (9) implies that $C_{12}(w, y) \geq 1$ or $C_{13}(w, y) \geq 1$ is violated. Since any $(w, y) \in D T_{S}(x)$ can be written as a convex combination of these graphs, there is always a fraction of the flow that cannot reach vertex 1 , proving the result.

The intuitive idea behind the distance transformation is that fractional solutions are often mapped to layered graphs where node splitting occurs, as in Figure 4. The node splitting then cuts some paths in the original graph which results in a decrease of connectivity. This decrease is often enough to cut the fractional solutions. Figure 4 shows that the node splitting breaks the cycle in all cases but the upper one. Nevertheless, the connectivity of the upper-left graph is also reduced because of the limited weight available on the directed edge linking the supervertices to the layered graph. When no node-splitting occurs, as in the left graph of Figure 5, graphs $G(x)$ and $\mathbf{G}(w, y)$ are isomorphic so that they satisfy the same connectivity requirements. It is therefore useful to be able to discover whether a given distance transformation leads to splitting of the fractional vectors. The result below partially answers this question by providing an approach to find out whether the graph induced by a given fractional solution $(x, w, y) \in P_{S}$ contains split nodes.

Proposition 4. Let $x \in F(0,1)$ and $(w, y) \in D T_{S}(x)$. Consider a set of $Q$ positive convex multipliers $\lambda$ such that

$$
(x, w, y)=\sum_{q=1}^{Q} \lambda^{q}\left(x^{q}, w^{q}, y^{q}\right),
$$

where $x^{q} \in\{0,1\}^{m}$ and $\left(w^{q}, y^{q}\right)=D T_{S}\left(x^{q}\right)$ for each $q=1, \ldots, Q$. Then, any node $i \in V$ is split $Q^{\prime}$ times in $\mathbf{G}(w, y)$, where $Q^{\prime} \in\{1, \ldots, Q\}$ corresponds to the number of different values in set

$$
\left\{\operatorname{dist}_{G\left(x^{q}\right)}(i, S), q=1, \ldots, Q\right\} .
$$

Proof. From equation (10), we have that $w_{i}^{l}=\sum_{q=1}^{Q} \lambda^{q} w_{i}^{q l}$. By definition of $D T_{S}$, we further have that $w_{i}^{q \operatorname{dist}_{G\left(x^{q}\right)}(i, S)}=1$ for each $q \in\{1, \ldots, Q\}$, so that

$$
w_{i}^{l}=\sum_{q: \operatorname{dist}_{G\left(x^{q}\right)}(i, S)=l} \lambda^{q},
$$

which is positive for each $l \in\{0, \ldots, n\}$ corresponding to a value in the set defined in (11).

Proposition 4 is an important result in understanding the structure of fractional vectors in $P_{S}$, which is the key to efficiently apply distance transformation to network design problems. In particular, the proposition shows that any node $i \in V$ corresponding to $(x, w, y)$ defined by equation (10) is split if and only if

$$
\operatorname{dist}_{G\left(x^{q}\right)}(i, S) \neq \operatorname{dist}_{G\left(x^{q^{\prime}}\right)}(i, S),
$$


for some $q \neq q^{\prime}$ in $\{1, \ldots, Q\}$. The difficulty of using the node splitting to cut a particular fractional solution $x$ is that we must ensure that the splitting occurs for all $(w, y) \in D T_{S}(x)$, which is a complex task in general. Nevertheless, for some very special cases it is possible to predict that splitting always occurs, see the result below.

Proposition 5. Consider the distance transformation defined by a unique root $S=\{i\}$ for some $i \in V$, let $x \in F(0,1)$, and consider a node $j \in V \backslash\{i\}$. If $x_{i j} \in(0,1)$, node $j$ is split in $\mathbf{G}(w, y)$ for all $(w, y) \in D T_{S}(x)$.

Proof. Let

$$
(x, w, y)=\sum_{q=1}^{Q} \lambda^{q}\left(x^{q}, w^{q}, y^{q}\right)
$$

be any vector in $P_{S}$. Because $x_{i j} \in(0,1)$, there exists $q$ and $q^{\prime}$ in $Q$ such that $x_{i j}^{q}=1$ and $x_{i j}^{q^{\prime}}=0$. Hence, (12) holds, yielding the result.

\section{Linear programming formulations for the DT.}

3.1. "Natural" formulation for $P_{S}$. So far we have been using abstract distance transformations based on the ideal polytope $P_{S}$. To use $D T$ in practice, we should have a linear formulation for $P_{S}$, providing its convex hull in the ideal case. Unfortunately, the complete description of $P_{S}$ is not easy to find. Below, we provide a polynomial formulation for $P_{S}$ which, although not completely describing $P_{S}$, leads to very good improvements in the linear programming relaxation of some network design problems with connectivity requirements. Let $\delta_{E}(S)=\{\{i, j\} \in E,|\{i, j\} \cap S|=1\}$ and $E^{\prime}=E \backslash \delta_{E}(S)$. The formulation below links the three groups of variables $x, w$, and $y$ using the following constraints:

$$
\begin{gathered}
w_{i}^{0}=1, \forall i \in S \\
\sum_{l=1}^{n} w_{i}^{l}=1, \forall i \in V \backslash S \\
y_{i j}^{01}=x_{i j}, \forall\{i, j\} \in \delta_{E}(S), i \in S \\
\sum_{l=1}^{n} y_{i j}^{l l}+\sum_{l=1}^{n-2}\left(y_{i j}^{l(l+1)}+y_{j i}^{l(l+1)}\right)=x_{i j}, \forall\{i, j\} \in E^{\prime} \\
y_{i j}^{01} \leq w_{j}^{1}, \forall\{i, j\} \in \delta_{E}(S), i \in S \\
y_{i j}^{11}+y_{i j}^{12} \leq w_{i}^{1}, \\
y_{i j}^{11}+y_{j i}^{12} \leq w_{j}^{1}, \quad \forall\{i, j\} \in E^{\prime} \\
y_{i j}^{l l}+y_{i j}^{l(l+1)}+y_{j i}^{(l-1) l} \leq w_{i}^{l}, \quad \forall\{i, j\} \in E^{\prime} ; l=2, \ldots, n-2 \\
y_{i j}^{l l}+y_{j i}^{l(l+1)}+y_{i j}^{(l-1) l} \leq w_{j}^{l}, \quad \\
y_{j i}^{(n-2)(n-1)}+y_{j i}^{(n-1)(n-1)} \leq w_{i}^{n-1}, \\
y_{i j}^{(n-2)(n-1)}+y_{i j}^{(n-1)(n-1)} \leq w_{j}^{n-1}, \\
w_{i}^{l} \leq \sum_{\{j, i\} \in E} y_{j i}^{(l-1) l}, \forall i \in V \backslash S ; l=2, \ldots, n-1 \\
0 \leq x, w, y \leq 1 .
\end{gathered}
$$

Constraints (13a-13b) state that each vertex should be in one of its possible levels. Constraints (13c-13h) state that each original edge variable $x_{i j}$ should be translated 


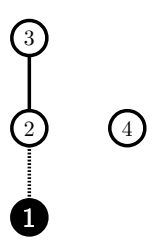

(a) $G(x)$

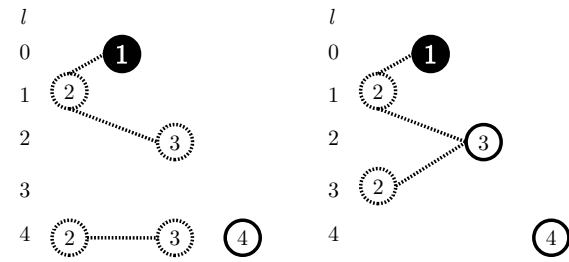

(b) $\mathbf{G}(w, y)(\mathrm{c}) \mathbf{G}(w, y)$ for some
for the unique $(w, y) \in \operatorname{ext}\left(D T_{S}^{F}(x)\right)$
$(w, y) \in D T_{S}(x)$

FIG. 6. Example of fractional $x$, the single point in $D T_{S}(x)$, and a point in $\operatorname{ext}\left(D T_{S}^{F}(x)\right)$ $(S=\{1\})$.

into a variable $y_{i j}^{l_{1} l_{2}}$ such that both $w_{i}^{l_{1}}$ and $w_{j}^{l_{2}}$ have value one. Constraints (13i) state that a vertex $i$ can only be in level $l<n$ if it is reached by at least one edge $\{j, i\}$ from level $(l-1)$. Let $P_{S}^{F}$ be the polytope defined by constraints (13). We prove below that the above constraints yield a valid formulation for polytope $P_{S}$ defined in (3).

Proposition 6. Linear constraints (13) yields a valid formulation for polytope $P_{S}$. Hence, given $x \in\{0,1\}^{m},(x, w, y) \in P_{S}^{F}$ if and only if $(w, y)=D T_{S}(x)$.

Proof. The validity of $(13 \mathrm{a}-13 \mathrm{j})$ follows from the observation that $\left(x, D T_{S}(x)\right)$ satisfies the constraints for each $x \in\{0,1\}^{m}$. To see that (13a-13j) is also a formulation for $P_{S}$, we consider a binary vector $x \in\{0,1\}^{m}$ and see by induction on $l$ that $P_{S}^{F}$ contains a unique solution where, for each node $i$ connected to $S$ in $G(x), w_{i}^{l}=1$ if and only if $i$ is at distance $l$ from $S$. If $i$ is not connected to $S$, the corresponding (13b) constraint ensures that $w_{i}^{n}=1$.

The above formulation enables us to extend $D T_{S}$ to fractional vectors as done in Section 2.1. The pendant of equation (4) for $P_{S}^{F}$ is

$$
D T_{S}^{F}(x)=\left\{(w, y) \mid(x, w, y) \in P_{S}^{F}\right\}
$$

and $P \subseteq P_{S}^{F}$ implies that $D T_{S}(x) \subseteq D T_{S}^{F}(x)$ for any $x \in[0,1]^{n}$. When $x$ is fractional, the example from Figure 6 shows that the inclusion can be strict. Namely, Figure 6(c) depicts the graph induced by a vector $(w, y) \in D T_{S}^{F}(x)$ that does not belong to $D T_{S}(x)$ because it cannot be obtained as the convex combination of binary vectors. In fact, for that example, $D T_{S}(x)$ is reduced to the singleton $(w, y)$ that induces the graph in Figure 6(b).

For any $x \in[0,1]^{m}$, one can also extend the connectivity requirements to the graphs induced by any $(w, y) \in D T_{S}^{F}(x)$. We define

$$
\mathcal{C}^{F_{S}}=\operatorname{Proj}_{x}\left\{(x, y, w) \in P_{S}^{F} \mid C_{u v}^{H}(w, y) \geq K, \forall(u, v) \in D\right\},
$$

and Proposition 2 can be completed with the following result.

Proposition 7. For any connectivity requirements constraints, it always holds that

$$
\mathcal{C}^{P_{S}} \subseteq \mathcal{C}^{F_{S}} \subseteq \mathcal{C}^{0}
$$




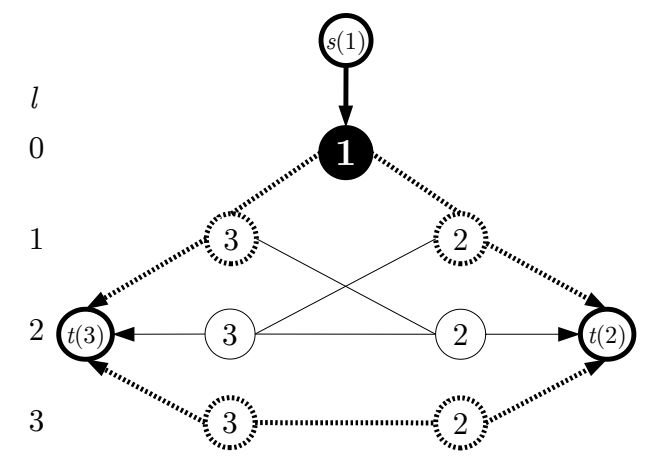

FIG. 7. Graph $\overline{\mathbf{G}}(w, y)=(\overline{\mathbf{V}}(w), \overline{\mathbf{E}}(w, y))$ associated to the second extreme point $(w, y)$ depicted in Figure 4 and considering demands $(1,2)$ and $(1,3)$. Thin edges (resp. nodes) correspond to the components of $y$ (resp. $w$ ) equal to zero and do not belong to $\overline{\mathbf{E}}(w, y)$ (resp. $\overline{\mathbf{V}}(w))$.

Proof. The inclusion $\mathcal{C}^{P_{S}} \subseteq \mathcal{C}^{F_{S}}$ follows from the fact that $P \subseteq P_{S}^{F}$. Then, constraints (13d) and (13f) enable us to prove inclusion $\mathcal{C}^{F_{S}} \subseteq \mathcal{C}^{0}$ by using the same flattening argument as the one used in the proof of Proposition 2.

We study next whether inclusions in (15) can be strict. We see easily that Proposition 3 extends to $\mathcal{C}^{F_{S}}$, providing an instance for which $\mathcal{C}^{F_{S}} \subset \mathcal{C}^{0}$. We then turn to inclusion $\mathcal{C}^{P_{S}} \subseteq \mathcal{C}^{F_{S}}$. The example from Figure 6 does not lead to strict inclusion because the graphs of Figures 6(b) and 6(c) satisfy the same connectivity requirements, namely $C_{23}^{1}(w, y) \geq 1$. However, we do have numerical evidence that the inclusion can be indeed strict, which is left out of the manuscript to simplify our exposure.

3.2. Limiting the levels. The DT described in the previous subsections can lead to large formulations. Due to the number of layers in graph $\mathbf{G}$, formulation (13) introduces $O(n m)$ variables and constraints. For some NDPs, we can eliminate most levels without affecting the transformation. For instance, if all demands in $D$ have a common extremity, and the connectivity requirements asks for paths bounded by some number $H$, then we can restrict the number of layers to $H+1$. This example arises in the survivable network design problems studied by [18].

However, it is possible to define DTs that use only a small number of layers $L$, regardless to the NDP under consideration. This decreases the size of the associated linear programming formulations, but may also decrease the node splitting, and thus, the gains in terms of gap reduction. In fact, there is a trade-off between the chosen value of $L$ and the quality of the DT. We suppose that $G(x)$ is the graph induced by some binary vector $x$ and that $\mathbf{G}_{L}$ is a graph that consists of $L$ layers. The truncated distance transformation $D T_{S}^{L}$ sets the image of node $i$ in $\mathbf{G}_{L}$ to layer $\min \left(\operatorname{dist}_{G(x)}(i, S), L-1\right)$. This means that levels from $L-1$ to $n-1$ of the original $\mathbf{G}$ are flattened into a single level $L-1$ in $\mathbf{G}_{L}$; nodes not connected to $S$ are still mapped to layer $L$. Edges are mapped subsequently according to the images of their extremities. This will not affect much the quality of the DT when few nodes $i$ have $\operatorname{dist}_{G(x)}(i, S) \geq L$ in typical solutions $x$. Formulation (13) is adapted for this modification by changing constraints $(13 \mathrm{~g}-13 \mathrm{i})$, reducing the size of the formulation to $O(L m)$ variables and constraints.

\section{Formulating the connectivity requirements.}


4.1. Simple connectivity requirements. In order to provide a linear programming formulation for an NDP, one still has to reformulate the connectivity requirements constraints with linear constraints. We start our approach with the simple constraints

$$
C_{u v}(w, y) \geq K, \quad \forall(u, v) \in D
$$

considered in the previous sections. Recall that constraint (16) impose that, for each $(u, v) \in D$, the value of the maximum flow between $s(u)$ and $t(v)$ in $\overline{\mathbf{G}}(w, y)$ be not smaller than $K$, see Figure 7 for an example of graph $\overline{\mathbf{G}}(w, y)=(\overline{\mathbf{V}}(w), \overline{\mathbf{E}}(w, y))$. We describe next how to express the constraints for a single demand $\{u, v\} \in D$ using either flow variables or cutset inequalities, and disregarding the level reductions discussed in the previous section. The flow formulation complements Formulation (13) with two flow variables $f_{i j}^{l_{1} l_{2}}$ and $f_{j i}^{l_{2} l_{1}}$ for each undirected edge $\left\{i^{l_{1}}, j^{l_{2}}\right\}$ in $\overline{\mathbf{G}}(w, y)$, and two flow variables $f_{s(u) u}^{l}$ and $f_{v t(v)}^{l}$ for each demand $(u, v) \in D$ and $l \in\{1, \ldots, n\}$ (notice that if $u$ belongs to $S$, we only introduce flow variable $f_{s(u) u}^{0}$, see Figure 7, and similarly if $v \in S$ ). Then, we impose that capacity be respected for all edges

$$
\begin{aligned}
f_{i j}^{l_{1} l_{2}}+f_{j i}^{l_{2} l_{1}} \leq y_{i j}^{l_{1} l_{2}}, & \forall\left\{i^{l_{1}}, j^{l_{2}}\right\} \in \mathbf{E} \\
f_{s(u) i}^{l} \leq K w_{i}^{l}, & \forall i^{l} \in \mathbf{V} \\
f_{i t(v)}^{l} & \leq K w_{i}^{l}, \quad \forall i^{l} \in \mathbf{V}
\end{aligned},
$$

flow be conserved for nodes in $\mathbf{V}$

$$
f_{s(u) i}^{l_{1}}-f_{i t(v)}^{l_{1}}+\sum_{\left\{i^{l_{1}}, j^{l_{2}}\right\} \in \delta_{\mathbf{E}}\left(i^{l_{1}}\right)}\left(f_{j i}^{l_{2} l_{1}}-f_{i j}^{l_{1} l_{2}}\right)=0, \forall i^{l_{1}} \in \mathbf{V},
$$

and that the flow exiting supervertex $s(u)$ exceeds $K$

$$
\begin{aligned}
f_{s(u) i}^{0} \geq K, & \text { if } u \in S, \\
\sum_{l=1}^{n} f_{s(u) i}^{l} \geq K, & \text { otherwise. }
\end{aligned}
$$

Alternatively to constraints (17-19), cutset inequalities impose that

$$
\sum_{l: u^{l} \in \mathbf{V} \backslash \mathbf{U}} K w_{u}^{l}+\sum_{l: v^{l} \in \mathbf{U}} K w_{v}^{l}+\sum_{\left\{i^{l_{1}}, j^{l_{2}}\right\} \in \delta_{\mathbf{E}}(\mathbf{U})} y_{i j}^{l_{1} l_{2}} \geq K, \forall \mathbf{U} \subseteq \mathbf{V} .
$$

We illustrate next cutset inequalities on an example based on the solution depicted in Figure 7 together with connectivity requirement $C_{12}(w, y) \geq 1$. If $\mathbf{U}=\left\{1^{0}, 2^{1}, 3^{1}\right\}$, then inequality (20) becomes

$$
w_{2}^{1}+y_{23}^{12}+y_{32}^{12} \geq 1
$$

which is violated by the solution depicted in Figure 7 .

4.2. Hop constraints. We study next the more complex connectivity requirements obtained by limiting the path length (hops) used to transmit the flow by a given integer $H$. Namely, we consider matrix $\mathfrak{C}(x) \in \mathbb{R}_{+}^{|D| \times(n-1)}$ with $\mathfrak{C}_{u v}^{H}(x)$ equal to the maximum flow between $u$ and $v$ in $G(x)$ using paths with at most $H$ hops. Similarly, we define matrix $\mathfrak{C}(w, y) \in \mathbb{R}_{+}^{|D| \times(n-1)}$ with $\mathfrak{C}_{u v}^{H}(w, y)$ equal to the maximum flow between the supervertices $s(u)$ and $t(v)$ in $\overline{\mathbf{G}}(w, y)$ using paths with at 


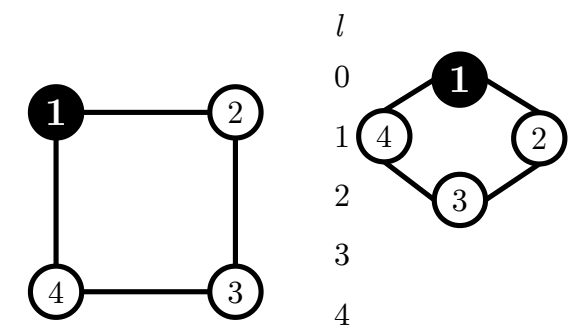

(a) Graph $G$.

(b) Graph G.

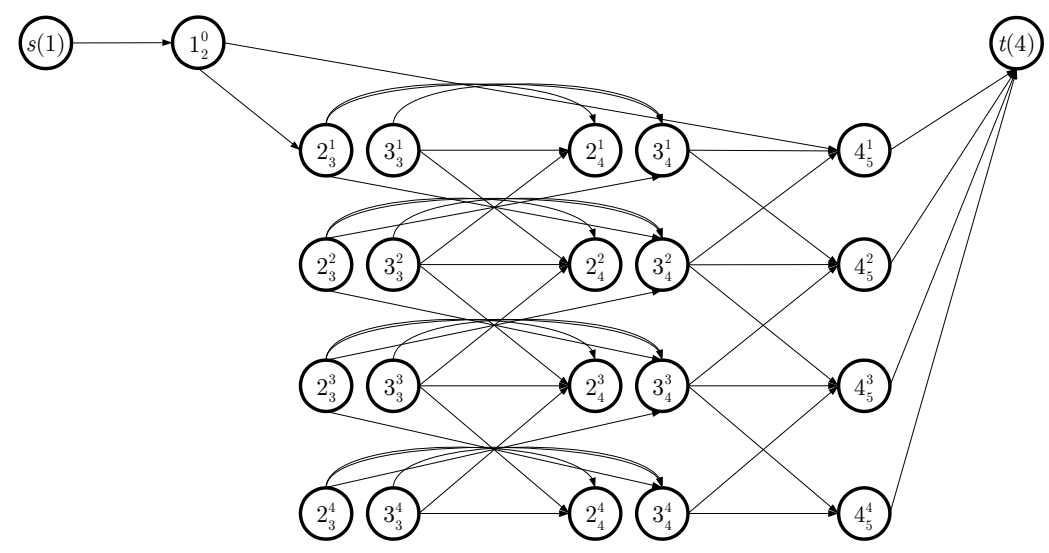

(c) Graph $\mathbf{G}^{14}$ for $H=3$.

FIG. 8. Hop-level graph.

most $H+2$ hops. With these definitions, we see immediately that $C(x)=\mathfrak{C}^{n-1}(x)$ and $C(w, y)=\mathfrak{C}^{n-1}(w, y)$. The counterparts of (5) and (16) for $\mathfrak{C}$ are

$$
\mathfrak{C}_{u v}^{H}(x) \geq K, \quad \forall(u, v) \in D
$$

and

$$
\mathfrak{C}_{u v}^{H}(w, y) \geq K, \quad \forall(u, v) \in D
$$

Remind that connectivity constraints (21) can be expressed by a hop-indexed flow formulation first introduced in [8]. The formulation has been extended to handle (22) in [18], denoted DT-HOP indexed flow formulation therein, and we recall it below. Given a demand $(u, v) \in D$ and an integer $H$, the formulation considers a directed layered graph $\mathbf{G}^{u v}=\left(\mathbf{V}^{u v}, \mathbf{A}^{u v}\right)$, where the definition of $\mathbf{V}^{u v}=\mathbf{V}_{1}^{u v} \cup \cdots \cup \mathbf{V}_{H+3}^{u v}$ depends on whether $\{u, v\}$ intersects $S$. If $\{u, v\} \cap S=\emptyset$, then $\mathbf{V}_{1}^{u v}=\{s(u)\}$, $\mathbf{V}_{2}^{u v}=\left\{u^{l} \mid 1 \leq l \leq n\right\}, \mathbf{V}_{h}^{u v}=\mathbf{V} \backslash\left\{\left\{u^{l}, v^{l}\right\} \mid 1 \leq l \leq n\right\}, h=3, \ldots, H+1$, $\mathbf{V}_{H+2}^{u v}=\left\{v^{l} \mid 1 \leq l \leq n\right\}$ and $\mathbf{V}_{H+3}^{u v}=\{t(v)\}$. If $u \in S$ or $v \in S$, we have instead $\mathbf{V}_{2}^{u v}=\left\{u^{0}\right\}$ or $\mathbf{V}_{H+2}^{u v}=\left\{v^{0}\right\}$, respectively, see Figure 8 for an example where $u \in S$ and $v \notin S$. Let $\mathbf{i}_{h}$ be the copy of $\mathbf{i} \in \mathbf{V}$ in the $h$-th layer of graph $\mathbf{G}^{u v}$, that is, $\mathbf{i}=i^{l}$ 
for some $i \in V, 0 \leq l \leq n$ and $\mathbf{i}_{h}=i_{h}^{l}$. The arcs set is defined by (see again Figure 8)

$$
\begin{aligned}
\mathbf{A}^{u v} & =\left\{\left(s(u), \mathbf{u}_{2}\right) \mid \mathbf{u}_{2} \in \mathbf{V}_{2}^{u v}\right\} \\
& \cup\left\{\left(\mathbf{u}_{2}, \mathbf{i}_{3}\right) \mid\{\mathbf{u}, \mathbf{i}\} \in \mathbf{E}, \mathbf{u}_{2} \in \mathbf{V}_{2}^{u v}, \mathbf{i}_{3} \in \mathbf{V}_{3}^{u v}\right\} \\
& \cup\left\{\left(\mathbf{i}_{h}, \mathbf{j}_{h+1}\right) \mid\{\mathbf{i}, \mathbf{j}\} \in \mathbf{E}, \mathbf{i}_{h} \in \mathbf{V}_{h}^{u v}, \mathbf{j}_{h+1} \in \mathbf{V}_{h+1}^{u v}, 3 \leq h \leq H\right\} \\
& \cup\left\{\left(\mathbf{i}_{h}, \mathbf{i}_{h+1}\right) \mid 3 \leq h \leq H, \mathbf{i}_{h} \in \mathbf{V}_{h}^{u v}\right\} \\
& \cup\left\{\left(\mathbf{i}_{H+1}, \mathbf{v}_{H+2}\right) \mid\{\mathbf{v}, \mathbf{i}\} \in \mathbf{E}, \mathbf{v}_{H+2} \in \mathbf{V}_{H+2}^{u v}, \mathbf{i}_{H+1} \in \mathbf{V}_{H+1}^{u v}\right\} \\
& \cup\left\{\left(\mathbf{v}_{H+2}, t(v)\right) \mid \mathbf{v}_{H+2} \in \mathbf{V}_{H+2}^{u v}\right\} \\
& \cup\left\{\left(\mathbf{u}_{2}, \mathbf{v}_{H+2}\right) \mid\{\mathbf{u}, \mathbf{v}\} \in \mathbf{E}, \mathbf{u}_{2} \in \mathbf{V}_{2}^{u v}, \mathbf{v}_{H+2} \in \mathbf{V}_{H+2}^{u v}\right\} .
\end{aligned}
$$

Given this auxiliary graph, the DT-HOP indexed flow formulation complements formulation (13) with a flow variable $g_{\mathbf{i} \mathbf{j}}^{u v, h}$ for each $\operatorname{arc}\left(\mathbf{i}_{h}, \mathbf{j}_{h+1}\right) \in \mathbf{A}^{u v}$. To simplify notations, we omit index $u v$ from the flow variables in what follows. Then, we impose that capacity be respected for all edges

$$
\begin{gathered}
g_{s(u) \mathbf{u}}^{1} \leq K w_{\mathbf{u}}, \forall \mathbf{u}_{2} \in \mathbf{V}_{2}^{u v} \\
g_{\mathbf{u i}}^{2} \leq y_{\mathbf{u i}}, \forall\{\mathbf{u}, \mathbf{i}\} \in \mathbf{E}, \mathbf{u}_{2} \in \mathbf{V}_{2}^{u v}, \mathbf{i}_{3} \in \mathbf{V}_{3}^{u v} \\
\sum_{h=3}^{H}\left(g_{\mathbf{i j}}^{h}+g_{\mathbf{j i}}^{h}\right) \leq y_{\mathbf{i j}}, \forall\{\mathbf{i}, \mathbf{j}\} \in \mathbf{E}, \mathbf{i}_{h} \in \mathbf{V}_{h}^{u v}, \mathbf{j}_{h+1} \in \mathbf{V}_{h+1}^{u v}, 3 \leq h \leq H \\
g_{\mathbf{i v}}^{H+1} \leq y_{\mathbf{v i}}, \forall\{\mathbf{v}, \mathbf{i}\} \in \mathbf{E}, \mathbf{v}_{H+2} \in \mathbf{V}_{H+2}^{u v}, \mathbf{i}_{H+1} \in \mathbf{V}_{H+1}^{u v} \\
g_{\mathbf{v} t(v)}^{H+2} \leq K w_{\mathbf{v}}, \forall \mathbf{v}_{H+2} \in \mathbf{V}_{H+2}^{u v} \\
g_{\mathbf{u v}} \leq y_{\mathbf{u v}}, \forall\{\mathbf{u}, \mathbf{v}\} \in \mathbf{E}, \mathbf{u}_{2} \in \mathbf{V}_{2}^{u v}, \mathbf{v}_{H+2} \in \mathbf{V}_{H+2}^{u v} .
\end{gathered}
$$

Arcs in (26) have an infinite capacity so that no capacity constraints are written for these arcs. The counterpart of flow conservations constraints in graph $\mathbf{G}^{u v}$ is readily obtained from (18). Finally, we need to impose that the flow exiting supervertex $s(u) \in \mathbf{V}_{1}^{u v}$ exceeds $K$ :

$$
\sum_{\mathbf{u}_{2} \in \mathbf{V}_{2}^{u v}} g_{s(u) \mathbf{u}_{2}}^{0} \geq K
$$

Capacity constraints (32) prevent the above system to define a pure network flow problem, having the integrality property. Hence, in general we need to impose integrality restrictions on $g$. However, in the cases $H=2,3$, one can readily extend the results from $[12,2]$ to show that the DT-HOP indexed formulation is indeed integral. Actually, for those cases it is possible avoid including the DT-HOP indexed variables and constraints in the Formulation (13) and replace them by cuts separated by the min-cut algorithm over $\mathbf{G}^{u v}$, as shown in the following example.

Consider graph $G=(V, E)$, defined by $V=\{1,2,3,4,5\}$ and $E=\{\{1,2\}$, $\{1,3\},\{2,3\},\{3,4\},\{3,5\},\{4,5\}\}$, let $\mathbf{G}=(\mathbf{V}, \mathbf{E})$ be the associated layered graph and Figure 9(a) represent a fractional solution that satisfies $\mathfrak{C}_{15}^{3}(x) \geq 1$. Figure $9(\mathrm{~b})$ represents the graph associated to some $(w, y) \in D T_{S}(x)$ that violates $\mathfrak{C}_{15}^{3}(w, y) \geq 1$. We show next how to separate a violated cut violated by $\mathbf{G}(w, y)$. Without loss of generality, we can restrict ourselves to the subsets of nodes and edges of $\mathbf{G}$ that belong to at least one graph of $\left\{\mathbf{G}\left(D T\left(x^{\prime}\right)\right): x^{\prime} \in\{0,1\}^{m}\right\}$, which are represented in Figure $9(\mathrm{c})$ and can be obtained automatically using preprocessing algorithms. The feasibility of $\mathfrak{C}_{15}^{3}(w, y) \geq 1$ is tested by looking for a feasible flow of one unit between 

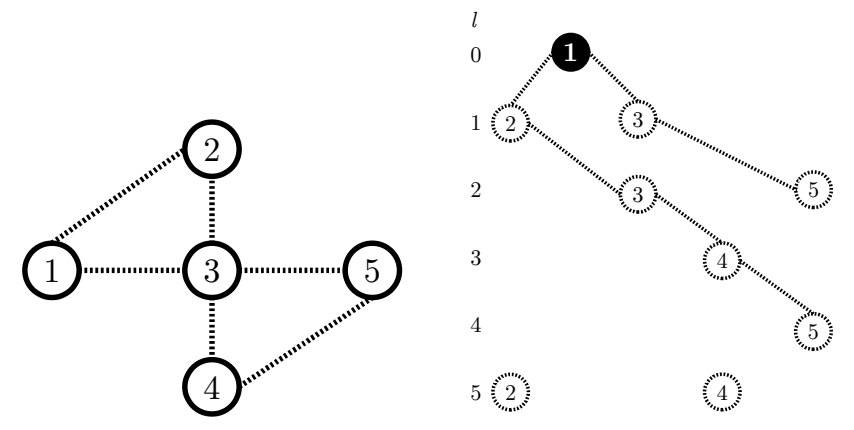

(a) Fractional solution $G(x)$ that satisfies $\mathfrak{C}_{15}^{3}(x) \geq 1$.
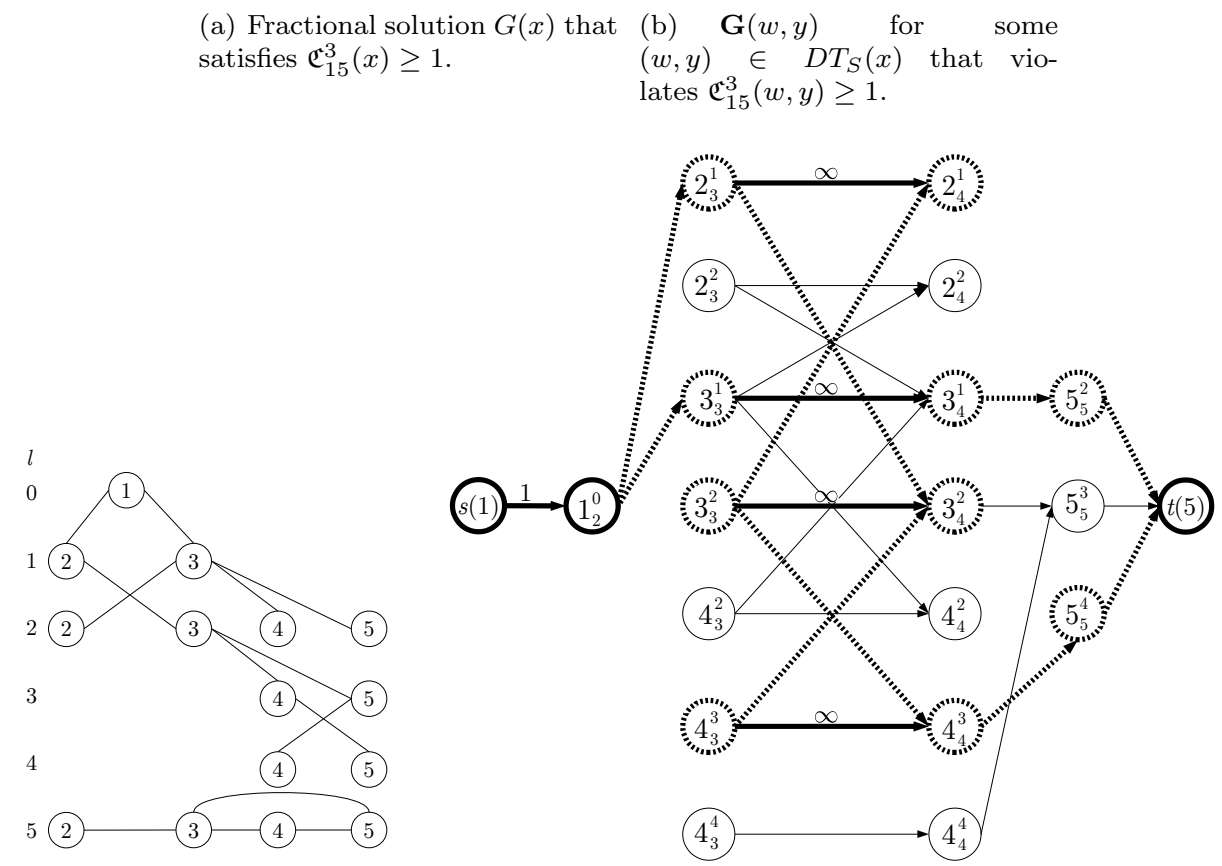

(c) $\bigcup_{x^{\prime} \in\{0,1\}^{m}} \mathbf{G}\left(D T_{S}\left(x^{\prime}\right)\right)$.

(d) Transformation of $\mathbf{G}(w, y)$ following Figure 1 from [13]. Thin nodes (resp. edges) correspond to the components of $w$ (resp. y) equal to zero. Nodes in level 5 are omitted because they are not connected to 1 .

FIG. 9. Separation of 3-path cut inequalities following [13].

$s(1)$ and $t(5)$ in the expanded graph depicted in Figure $9(\mathrm{~d})$. Looking for a cut of minimum capacity that contains $s(1)$, we obtain either inequality

$$
y_{13}^{01}+y_{35}^{23} \geq 1,
$$

or inequality

$$
w_{5}^{2}+y_{35}^{23} \geq 1,
$$

which are both violated by the solution depicted in Figure 9(b). Those inequalities correspond to the counterparts of the 3 -path-cut inequalities proposed in [12, 2].

Whenever $H \geq 4$, the DT-HOP indexed flow formulation cannot be replaced by inequalities obtained by the min-cut algorithm. However, it can be numerically 
efficient to avoid the inclusion of the formulation and replace it by Benders inequalities for variables $y$ and $w$, similarly as [3].

\section{Numerical experiments for the DT.}

5.1. The Steiner Forest Problem. The SFP has the following natural formulation:

$$
\begin{array}{ll}
\text { Min } & \sum_{e \in E} c_{e} x_{e} \\
\text { S.t. } & \sum_{e \in \delta_{E}(S)} x_{e} \geq 1 \forall(u, v) \in D ; \forall S \subset V, u \in S, v \notin S \\
& x_{e} \text { binary } \forall e \in E .
\end{array}
$$

Constraints (37b) are known as undirected cut inequalities. This formulation is the basis for the classical primal-dual 2-approximated algorithm for the SFP [7]. Nevertheless, (37) does not provide effective exact branch-and-cut algorithms, duality gaps of more than $20 \%$ are typical on practical instances. For example, consider an instance defined over a complete graph with vertices $\{1,2,3\}$ and $D=\{(1,2),(1,3)\}$. The fractional solution $x_{12}=x_{13}=x_{23}=0.5$ mentioned previously satisfies all constraints (37b).

The difficulty of devising a directed formulation for SFP lies in the fact that it is not known beforehand which demands will belong to the same connected component (a subtree) of an optimal solution. A strong SFP formulation was proposed by Magnanti and Raghavan [17], based on the concept of consistent edge orientations across demands. In that concept, each connected component is represented by a directed tree rooted at the first vertex of the demand with smaller index in the component. However, the formulation in [17] is not much practical since it contains an exponential number of constraints and no polynomial algorithm for separating them is known. The lifted-cut formulation proposed by Konemann et al. [15] can be stronger than (37) and its linear relaxation can be solved in polynomial time. Recently, Schmidt et al. [22] presented four new formulations (essentially three formulations, since two of them are equivalent). The strongest new formulations are based on the same consistent edge orientations across demands concept from [17], however, their linear relaxations can be solved in polynomial time.

Figure 10 depicts a small STF instance with 8 vertices and 12 edges considered in [22], edge costs are unitary and the 4 demands are represented by pairs of identical symbols. The optimal solution value is 7 . Table 1 presents the linear relaxation values for each existing formulation (taken from [22]) and also for the new formulation obtained by applying the DT to the natural formulation.

This small example illustrates the potential power of the DT on STF. In fact, it proves that the weak natural formulation is transformed into a new formulation that can be strictly stronger than any other known STF formulation in some instances. On the other hand, the result obtained by setting $L=3$ shows that the limitation of levels, necessary on larger instances, may affect the linear relaxation bounds significantly.

The following experiments were performed in a single core of a machine with processor i7 at $2.5 \mathrm{GHz}$ and $16 \mathrm{~GB}$ of RAM. The tested formulations were implemented over the XPRESS-Optimizer 7.3. We performed tests with 3 types of instances:

- Small STF instances (pdh, di-yuan, dfn-gwin, polska and nobel-us) available in the SNDLib. 


\begin{tabular}{lr}
\hline Formulation & Linear Relaxation \\
\hline Natural (37) & 4 \\
Lifted-cut [15] & 4 \\
Full directed flow based [17] & 6 \\
Tree-based [22] & 5 \\
Extended cut-based [22] & 5.14 \\
Strengthened extended cut-based [22] & 6 \\
\hline Distance Transformation & 7 \\
Distance Transformation L=3 & 5 \\
\hline \multicolumn{2}{c}{ TABLE 1 } \\
\multicolumn{2}{c}{ Linear relaxation value for instance in Figure 10 }
\end{tabular}

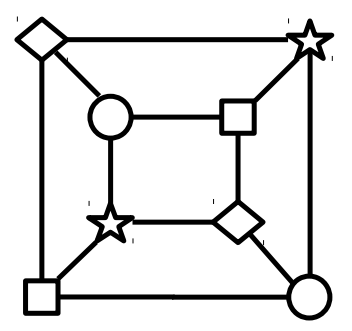

FIG. 10. STF instance with 4 demands (pairs of identical symbols), unitary edge costs.

- Steiner instances C01_st,...,C10_st from the SteinLib. Those instances are defined over random graphs with 500 vertices. For an instance with terminalset $T$, we defined $D$ as $\{(r, v): v \in T, v \neq r\}$, where $r$ is the terminal with smaller index. Those instances can be easily solved by SPG codes using a directed formulation. Nevertheless, it is interesting to see how the DT can improve the undirected formulation.

- SPF instances $\mathrm{C} 01, \ldots, \mathrm{C} 10$ derived from the above instances as follows. The set $D$ is obtained by pairing consecutive terminals in $T$. If $|T|$ is odd, an extra demand from the first to the last terminal is included. To the best of our knowledge, some of those instances can be very hard for current solution methods.

Table 2 compares gaps (with respect to optimal or best known UBs) and times to solve linear relaxations for: (1) natural formulation (37); (2) Lifted-cut formulation [15]; (3) Strengthened Extended Cut-Based (SECB) [22] ; (4) DT reformulation over the natural formulation, for $L=3, L=4$, and $L=5$, using unitary distances and with a singleton set $S$ containing the first terminal. Some comments on those results:

- The lifted-cut formulation gaps are not much better than those from the natural formulation.

- The SECB formulation assumes that demands with a common vertex will be merged into demands containing more than two vertices. The connectivity requirement of those demands is the existence of paths joining all its vertices. In all SNDLib and C_st instances this preprocessing reduces all demands into a single demand. The SECB formulation for a single demand containing several vertices is exactly equivalent to the directed Steiner formulation. So, it is expected that the gap for those types is very close zero and the times small. The demands are not merged on the $\mathrm{C}$ instances, so the test of the SECB formulation is significant for that type. The experiments show that the 
gaps obtained are small, however the number of variables in the formulation $(O(m .|D|)$ and the number of times that the min-cut separation algorithm has to be called per separation round $\left(O\left(|D|^{2}\right)\right.$ makes the formulation slow when $|D|$ grows. In 5 of the $\mathrm{C}$ instances the solution was stopped at 1800 seconds, before the cut separation converged.

- The DT can reduce the gaps significantly with respect to the natural formulation. As expected, the average gaps decrease when $L$ increase, but the improvement quickly becomes marginal. The DT with $L=3$ seems to be the best compromise between gap and running times on most instances. While the gap reductions are remarkable for SNDLib and C_st instances, they are less impressive for the $\mathrm{C}$ instances. We verified that their distance transformed fractional solutions were divided into a number of connected components. All those components, except the one that contained the vertex in $S$, are in level $L$, where vertex splittings do not happen.

In order to make the DT effective on that last case, we devised an iterative scheme for choosing a larger set $S$. We start with a single vertex in $S$ and solve the linear relaxation of the corresponding DT. While the fractional solution still contains vertices in level $L$, we introduce one vertex from the larger connected component in $L$ and solve the new DT again. Table 3 shows the results of this dynamic procedure for $L=3, L=4$, and $L=5$. While the resulting gaps are quite better, they are still large when compared with those obtained in other types of instances.

Finally, Table 4 compares the results of the full branch-and-cut over the original formulation (37) with the branch-and-cut over the DT reformulation, for some chosen parameterization. We mark in bold the time of the method that could solve the instance faster. If no method could solve the instance, either because the time limit of 7200 seconds was exceeded or because it went out of memory, we mark in bold the smallest final gap obtained. We did not passed any external upper bound to the branch-and-cut, those gaps are with respect to the best solution found by the method itself. Although the harder instances could not be solved to optimality, it is clear that the overall performance of the DT reformulation is much better. 


\begin{tabular}{|c|c|c|c|c|c|c|c|c|c|c|c|c|c|c|c|c|}
\hline \multirow[b]{2}{*}{ Instance } & \multirow[b]{2}{*}{$|V|$} & \multirow[b]{2}{*}{$|E|$} & \multirow[b]{2}{*}{$|D|$} & \multirow[b]{2}{*}{ UB } & \multicolumn{2}{|c|}{ Natural } & \multicolumn{2}{|c|}{ Lifted-cut } & \multicolumn{2}{|c|}{ SECB } & \multicolumn{2}{|c|}{ DT L=3 } & \multicolumn{2}{|c|}{$\mathrm{DT} \mathrm{L}=4$} & \multicolumn{2}{|c|}{$\mathrm{DT} \mathrm{L}=5$} \\
\hline & & & & & $\begin{array}{l}\text { Gap } \\
(\%)\end{array}$ & $\begin{array}{l}\mathrm{T} \\
(\mathrm{s})\end{array}$ & $\begin{array}{l}\text { Gap } \\
(\%)\end{array}$ & $\begin{array}{l}\mathrm{T} \\
(\mathrm{s})\end{array}$ & $\begin{array}{l}\text { Gap } \\
(\%)\end{array}$ & $\mathrm{T}(\mathrm{s})$ & $\begin{array}{l}\text { Gap } \\
(\%)\end{array}$ & $\mathrm{T}(\mathrm{s})$ & $\begin{array}{l}\text { Gap } \\
(\%)\end{array}$ & $\mathrm{T}(\mathrm{s})$ & $\begin{array}{l}\text { Gap } \\
(\%)\end{array}$ & $\begin{array}{l}\mathrm{T} \\
(\mathrm{s})\end{array}$ \\
\hline pdh & 11 & 34 & 27 & 897551 & 41.0 & 0.00 & 32.2 & 0.35 & & & 0.0 & 0.01 & 0.0 & 0.01 & 0.0 & 0.01 \\
\hline di-yuan & 11 & 42 & 48 & 21570000 & 32.8 & 0.00 & 26.1 & 0.01 & & & 0.6 & 0.02 & 0.0 & 0.01 & 0.0 & 0.01 \\
\hline dfn-gwin & 11 & 47 & 9 & 79960 & 29.8 & 0.00 & 28.5 & 0.00 & & & 1.0 & 0.04 & 0.9 & 0.10 & 0.9 & 0.11 \\
\hline polska & 12 & 18 & 17 & 214100 & 38.1 & 0.00 & 29.2 & 0.01 & & & 0.0 & 0.01 & 0.0 & 0.01 & 0.0 & 0.01 \\
\hline nobel-us & 14 & 21 & 33 & 8481000 & 30.5 & 0.00 & 20.1 & 0.01 & & & 1.2 & 0.02 & 1.2 & 0.04 & 0.8 & 0.16 \\
\hline Avg. & & & & & 34.4 & 0.00 & 27.2 & 0.08 & & & 0.6 & 0.02 & 0.3 & 0.03 & 0.3 & 0.06 \\
\hline c01_st & 500 & 625 & 4 & 85 & 16.5 & 0.03 & 12.4 & 0.07 & & & 0.0 & 1.38 & 0.0 & 2.05 & 0.0 & 3.77 \\
\hline c02_st & 500 & 625 & 9 & 144 & 24.7 & 0.16 & 24.7 & 0.21 & & & 0.0 & 3.37 & 0.0 & 3.63 & 0.0 & 6.34 \\
\hline c03_st & 500 & 625 & 82 & 754 & 23.1 & 0.27 & 20.6 & 1.27 & & & 0.8 & 2.10 & 0.8 & 4.19 & 0.8 & 7.26 \\
\hline c04_st & 500 & 625 & 125 & 1079 & 20.8 & 0.29 & 19.6 & 2.40 & & & 0.1 & 1.09 & 0.1 & 6.32 & 0.1 & 5.99 \\
\hline c05_st & 500 & 625 & 249 & 1579 & 19.5 & 0.22 & 18.6 & 2.07 & & & 0.3 & 1.58 & 0.3 & 7.88 & 0.3 & 12.29 \\
\hline c06_st & 500 & 1000 & 4 & 55 & 17.3 & 0.16 & 14.6 & 0.28 & & & 4.2 & 4.00 & 3.6 & 10.10 & 4.2 & 8.89 \\
\hline c07_st & 500 & 1000 & 9 & 102 & 18.6 & 0.18 & 14.2 & 0.44 & & & 0.0 & 1.26 & 0.0 & 1.69 & 0.0 & 1.79 \\
\hline c08_st & 500 & 1000 & 82 & 509 & 25.5 & 0.29 & 24.3 & 1.27 & & & 0.1 & 4.73 & 0.1 & 13.65 & 0.1 & 27.45 \\
\hline c09_st & 500 & 1000 & 124 & 707 & 28.3 & 0.59 & 26.9 & 3.40 & & & 0.6 & 14.70 & 0.6 & 11.48 & 0.6 & 21.30 \\
\hline c10_st & 500 & 1000 & 249 & 1093 & 25.4 & 0.59 & 24.4 & 5.11 & & & 0.0 & 3.22 & 0.0 & 5.26 & 0.0 & 13.90 \\
\hline Avg. & & & & & 21.7 & 0.26 & 19.3 & 1.60 & & & 1.0 & 3.92 & 0.9 & 7.06 & 0.9 & 12.60 \\
\hline $\mathrm{c} 01$ & 500 & 625 & 3 & 85 & 16.5 & 0.03 & 5.3 & 0.11 & 0.6 & 15.6 & 1.0 & 3.53 & 1.0 & 4.62 & 1.0 & 7.34 \\
\hline c02 & 500 & 625 & 5 & 143 & 24.1 & 0.19 & 18.2 & 0.31 & 0.4 & 23.4 & 17.5 & 2.84 & 17.5 & 3.03 & 17.5 & 3.84 \\
\hline c03 & 500 & 625 & 42 & 754 & 23.1 & 0.39 & 21.6 & 0.95 & 0.1 & 623.7 & 18.9 & 13.80 & 18.9 & 21.30 & 18.9 & 27.40 \\
\hline $\mathrm{c} 04$ & 500 & 625 & 63 & 1079 & 20.8 & 0.57 & 19.3 & 1.06 & 0.8 & 1800 & 11.0 & 23.70 & 11.0 & 47.80 & 11.0 & 86.70 \\
\hline $\mathrm{c} 05$ & 500 & 625 & 125 & 1579 & 19.6 & 4.32 & 18.7 & 1.36 & 8.0 & 1800 & 13.9 & 82.60 & 13.9 & 71.00 & 13.9 & 134.80 \\
\hline c06 & 500 & 1000 & 3 & 47 & 3.2 & 0.13 & 3.2 & 0.38 & 0.0 & 1.43 & 0.0 & 2.83 & 0.0 & 2.91 & 0.0 & 4.44 \\
\hline $\mathrm{c} 07$ & 500 & 1000 & 5 & 89 & 10.1 & 0.07 & 8.4 & 0.32 & 0.0 & 19.1 & 0.0 & 2.70 & 0.0 & 2.51 & 0.0 & 2.98 \\
\hline $\mathrm{c} 08$ & 500 & 1000 & 42 & 509 & 25.5 & 0.77 & 25.3 & 0.92 & 4.2 & 1800 & 18.7 & 59.40 & 18.7 & 111.80 & 18.7 & 236.20 \\
\hline c09 & 500 & 1000 & 63 & 707 & 28.3 & 1.20 & 27.2 & 2.16 & 3.9 & 1800 & 23.0 & 39.80 & 23.0 & 122.20 & 23.0 & 276.60 \\
\hline c10 & 500 & 1000 & 125 & 1093 & 25.4 & 1.38 & 24.4 & 2.57 & 12.6 & 1800 & 20.3 & 113.90 & 20.3 & 228.40 & 20.2 & 617.90 \\
\hline Avg. & & & & & 18.5 & 0.84 & 16.1 & 1.00 & 3.1 & 968.3 & 11.4 & 31.70 & 11.3 & 56.40 & 11.3 & 127.90 \\
\hline Avg. & & & & & 22.7 & 0.35 & 19.5 & 1.08 & & & 5.2 & 4.47 & 5.0 & 8.31 & 5.0 & 14.80 \\
\hline
\end{tabular}

Natural [1], Lifted-cut [15], Strengthened Extended Cut-Based [22] and DT reformulation $(|S|=1)$ root gaps. 


\begin{tabular}{|c|c|c|c|c|c|c|}
\hline Inst & $\begin{array}{c}\mathrm{L}=3 \\
\operatorname{Gap}(\%)\end{array}$ & $\mathrm{T}(\mathrm{s})$ & $\begin{array}{c}\mathrm{L}=4 \\
\operatorname{Gap}(\%)\end{array}$ & $\mathrm{T}(\mathrm{s})$ & $\begin{array}{c}\mathrm{L}=5 \\
\operatorname{Gap}(\%)\end{array}$ & $\mathrm{T}(\mathrm{s})$ \\
\hline $\mathrm{c} 01$ & 0.98 & 3.53 & 0.98 & 4.62 & 0.98 & 7.34 \\
\hline $\mathrm{c} 02$ & 6.29 & 2.99 & 5.83 & 4.73 & 5.60 & 8.36 \\
\hline $\mathrm{c} 03$ & 7.36 & 6.86 & 7.05 & 20.84 & 6.97 & 28.86 \\
\hline $\mathrm{c04}$ & 4.50 & 10.64 & 4.32 & 25.12 & 4.20 & 31.95 \\
\hline $\mathrm{c} 05$ & 3.17 & 8.82 & 3.09 & 52.4 & 2.98 & 37.8 \\
\hline $\mathrm{c} 06$ & 0.00 & 2.83 & 0.00 & 2.91 & 0.00 & 4.44 \\
\hline $\mathrm{c} 07$ & 0.00 & 2.7 & 0.00 & 2.51 & 0.00 & 2.98 \\
\hline $\mathrm{c} 08$ & 9.89 & 20.16 & 9.69 & 40.14 & 9.57 & 62.45 \\
\hline c09 & 11.33 & 28.15 & 10.84 & 61.33 & 10.83 & 98.65 \\
\hline $\mathrm{c} 10$ & 8.12 & 40.38 & 7.88 & 82.15 & 7.78 & 135.88 \\
\hline Avg. & 4.86 & 11.96 & 4.52 & 27.49 & 4.45 & 38.89 \\
\hline
\end{tabular}

Root gaps for the dynamic choice of $S$ in $D T$.

\begin{tabular}{l|ccc|cccc}
\hline Inst & Nodes & Gap(\%) & $\mathrm{T}$ & Nodes & Gap(\%) & $\mathrm{T}(\mathrm{s})$ & \\
\hline pdh & 20183 & 0.00 & 10.52 & 1 & 0.00 & $\mathbf{0 . 0 1}$ & $\mathrm{L}=4|S|=1$ \\
di-yuan & 236 & 0.00 & 0.19 & 1 & 0.00 & $\mathbf{0 . 0 1}$ & $\mathrm{L}=4|S|=1$ \\
dfn-gwin & 1389 & 0.00 & 0.64 & 5 & 0.00 & $\mathbf{0 . 1 0}$ & $\mathrm{L}=4|S|=1$ \\
polska & 332 & 0.00 & 0.15 & 1 & 0.00 & $\mathbf{0 . 0 1}$ & $\mathrm{L}=4|S|=1$ \\
nobel-us & 186 & 0.00 & 0.09 & 3 & 0.00 & $\mathbf{0 . 0 5}$ & $\mathrm{L}=4|S|=1$ \\
\hline c01_st & 52 & 0.00 & $\mathbf{2 . 1 7}$ & 2 & 0.00 & 2.65 & $\mathrm{~L}=3|S|=1$ \\
c02_st & 4275 & 0.00 & 60.52 & 1 & 0.00 & $\mathbf{4 . 5 6}$ & $\mathrm{L}=4|S|=1$ \\
c03_st & 10887 & 28.38 & o.m. & 1212 & $\mathbf{0 . 5 3}$ & 7200 & $\mathrm{~L}=7|S|=1$ \\
c04_st & 20979 & 22.89 & 7200 & 255 & 0.00 & $\mathbf{6 . 1 8}$ & $\mathrm{L}=7|S|=1$ \\
c05_st & 45310 & 20.52 & 7200 & 149 & $\mathbf{0 . 0 6}$ & 7200 & $\mathrm{~L}=7|S|=1$ \\
c06_st & 241 & 0.00 & $\mathbf{5 . 4 8}$ & 14 & 0.00 & 10.77 & $\mathrm{~L}=3|S|=1$ \\
c07_st & 11409 & 0.00 & 565.24 & 1 & 0.00 & $\mathbf{1 . 5 5}$ & $\mathrm{L}=3|S|=1$ \\
c08_st & 12700 & 42.44 & o.m. & 12 & 0.00 & $\mathbf{3 0 . 7 4}$ & $\mathrm{L}=3|S|=1$ \\
c09_st & 16270 & 43.85 & o.m. & 514 & $\mathbf{0 . 3 2}$ & 7200 & $\mathrm{~L}=3|S|=1$ \\
c10_st & 29232 & 46.02 & 7200 & 20 & 0.00 & $\mathbf{2 9 2 . 5 8}$ & $\mathrm{L}=4|S|=1$ \\
\hline c01 & 56 & 0.00 & $\mathbf{2 . 1 3}$ & 3 & 0.00 & 3.38 & $\mathrm{~L}=3|S|=1$ \\
c02 & 4942 & 0.00 & 84.54 & 69 & 0.00 & $\mathbf{4 1 . 1 3}$ & $\mathrm{L}=4|S|=2$ \\
c03 & 18113 & 28.51 & 7200 & 119 & $\mathbf{1 0 . 6 1}$ & 7200 & $\mathrm{~L}=7|S|=8$ \\
c04 & 29877 & 23.91 & o.m. & 821 & $\mathbf{3 . 9 9}$ & 7200 & $\mathrm{~L}=3|S|=11$ \\
c05 & 34645 & 21.47 & 7200 & 198 & $\mathbf{3 . 0 4}$ & 7200 & $\mathrm{~L}=4|S|=9$ \\
c06 & 3 & 0.00 & $\mathbf{1 . 1 2}$ & 1 & 0.00 & 2.39 & $\mathrm{~L}=3|S|=1$ \\
c07 & 239 & 0.00 & 10.59 & 2 & 0.00 & $\mathbf{2 . 3 3}$ & $\mathrm{L}=3|S|=1$ \\
c08 & 12312 & 41.06 & o.m. & 439 & $\mathbf{8 . 8 4}$ & 7200 & $\mathrm{~L}=4|S|=13$ \\
c09 & 15243 & 48.37 & o.m. & 235 & $\mathbf{1 1 . 0}$ & 7200 & $\mathrm{~L}=3|S|=16$ \\
c10 & 17361 & 44.28 & 7200 & 17 & $\mathbf{7 . 4 1}$ & 7200 & $\mathrm{~L}=4|S|=27$ \\
\hline & & & $\mathrm{TABLE}$ & 4 & & & \\
& & & & &
\end{tabular}

Comparison of full branch-and-cut over natural formulation and over DT reformulation. Gaps are given with respect to the UBs found by branch-and-cut itself, not to the best known solutions.

5.2. The Survivable Network Design with Hop Constraints Problem. We consider in this section the HSNDP that has been defined in the introduction of the paper. We address the connectivity requirements by using the layered flow formulation described in Section 4.2, which results in a large extended formulation for the problem. It can be verified that using the flow formulations proposed in Section 4.2 
to model the connectivity requirements of HSNDP results in a formulation that is equivalent to the one originally proposed in [18]. Our objective in the section is to provide numerical evidence that the formulation is well-suited for Benders decomposition. Specifically, we compare the following three approaches for solving the extended formulation. First, we feed the formulation directly into CPLEX, leaving all parameters to their default values. Second, we consider the automatic Benders decomposition algorithm implemented in CPLEX 12.8, which decomposes the extended formulation into a master problem, that contains only the design variables $x, w$ and $y$ and Benders cuts, and one subproblem for each demand $D$ that contains the flow variables $g$ associated to that demand together with the flow conservations constraints and capacity constraints described in Section 4.2. Last, we implement an ad-hoc Benders decomposition algorithm using Callbacks and following closely the lines of [3]. Specifically, our algorithm solves the master problem through a branch-and-cut algorithm. Every time an integer solution $(\bar{x}, \bar{w}, \bar{y})$ is found in the branch-and-cut tree, all subproblems are solved to see if the solution $(\bar{x}, \bar{w}, \bar{y})$ is feasible for the original problem. If this is not the case, the subproblems return one or more Benders cuts that are added to the master problem at all nodes of the branch-and-cut tree.

All algorithms are we fed the solver with the best known solution (denoted BKS in Table 5) and allow it to fathom any node worse than BKS. Table 5 reports the results of our computational experiments. The table also contains the results obtained by using the classical layered formulations from [3] (denoted HOP formulation in the table) where the connectivity requirements are imposed through flows on layered graphs that are built directly on the original graph $G$. Hence, the HOP formulations contrasts with the the HOP-level formulation that models flows on layered graphs that are built on the top of the layered graph $\mathbf{G}$. 


\begin{tabular}{|c|c|c|c|c|c|c|c|c|c|c|c|c|c|c|c|c|c|c|}
\hline & & & & & & & & Cor & pact & & & Bende & s auto & & Benc & ders $\mathrm{v}$ & Callb & acks \\
\hline & & $\mathrm{H}$ & K & BKS & $\begin{array}{l}\text { root } \\
\text { LB }\end{array}$ & $\begin{array}{l}\text { root } \\
\text { gap }(\%)\end{array}$ & $\begin{array}{l}\text { final } \\
\text { LB }\end{array}$ & $\begin{array}{l}\text { final } \\
\text { UB }\end{array}$ & nodes & $\begin{array}{l}\text { Time } \\
\text { (s) }\end{array}$ & $\begin{array}{l}\text { final } \\
\text { LB }\end{array}$ & $\begin{array}{l}\text { final } \\
\text { UB }\end{array}$ & nodes & $\begin{array}{l}\text { Time } \\
\text { (s) }\end{array}$ & $\begin{array}{l}\text { final } \\
\text { LB }\end{array}$ & $\begin{array}{l}\text { final } \\
\text { UB }\end{array}$ & nodes & $\begin{array}{l}\text { Time } \\
\text { (s) }\end{array}$ \\
\hline & TC-20 & 3 & 2 & 607 & 495.1 & 18.4 & 607.0 & 607 & $67 K$ & 2353 & 607.0 & 607 & $3.6 M$ & 10748 & 607.0 & 607 & $973 K$ & 9294 \\
\hline & & 3 & 3 & 842 & 769.1 & 8.7 & 842.0 & 842 & $23 K$ & 1068 & 842.0 & 841 & $665 K$ & 1835 & 842.0 & 842 & $246 K$ & 1253 \\
\hline & & 4 & 2 & 536 & 442.3 & 17.5 & 506.5 & & $57 K$ & 36000 & 508.4 & & $2.8 M$ & 36000 & 523.0 & & $2.4 M$ & 36000 \\
\hline & & 4 & 3 & 750 & 699.7 & 6.7 & 750.0 & 750 & $16 K$ & 8776 & 750.0 & 750 & $421 K$ & 1530 & 750.0 & 750 & $286 K$ & 2469 \\
\hline & TE-20 & 3 & 2 & 776 & 603.1 & 22.3 & 776.0 & 776 & $59 K$ & 3508 & 724.4 & & $6.2 M$ & 36000 & 745.0 & & $1.5 \mathrm{M}$ & 36000 \\
\hline & & 3 & 3 & 1082 & 911.6 & 15.8 & 1082.0 & 1082 & $184 K$ & 9733 & 1011.5 & & $3.8 M$ & 36000 & 1024.9 & & $1.2 M$ & 36000 \\
\hline & & 4 & 2 & 670 & 516.9 & 22.8 & 587.5 & & $47 K$ & 36000 & 569.1 & & $1.6 \mathrm{M}$ & 36000 & 573.2 & & $720 \mathrm{~K}$ & 36000 \\
\hline & & 4 & 3 & 919 & 793.0 & 13.7 & 847.2 & & $44 K$ & 36000 & 830.4 & & $675 K$ & 36000 & 838.0 & & $558 K$ & 36000 \\
\hline 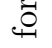 & TC-40 & 3 & 2 & 632 & 516.9 & 18.2 & 623.1 & 632 & $136 K$ & 36000 & 593.9 & & $3.6 M$ & 36000 & 592.2 & & $472 K$ & 36000 \\
\hline$\Rightarrow$ & & 3 & 3 & 889 & 795.0 & 10.6 & 881.5 & 889 & $127 K$ & 36000 & 856.4 & & $2.9 M$ & 36000 & 853.6 & & $489 K$ & 36000 \\
\hline & & 4 & 2 & 538 & 456.4 & 15.2 & 476.4 & & $1.6 \mathrm{~K}$ & 36000 & 501.9 & & $2.2 M$ & 36000 & 494.0 & & $341 K$ & 36000 \\
\hline & & 4 & 3 & 795 & 710.4 & 10.6 & 728.8 & & $1.8 K$ & 36000 & 747.8 & & $2.3 M$ & 36000 & 747.4 & & $437 K$ & 36000 \\
\hline & TE-40 & 3 & 2 & 790 & 616.9 & 21.9 & 746.7 & & $37 K$ & 36000 & 704.9 & & $2.1 M$ & 36000 & 695.3 & & $260 K$ & 36000 \\
\hline & & 3 & 3 & 1094 & 932.5 & 14.8 & 1045.0 & & $33 K$ & 36000 & 1009.5 & & $2.0 \mathrm{M}$ & 36000 & 999.4 & & $319 K$ & 36000 \\
\hline & & 4 & 2 & 661 & 528.4 & 20.1 & 546.4 & & 676 & 36000 & 568.2 & & $1.5 \mathrm{M}$ & 36000 & 558.5 & & $68 K$ & 36000 \\
\hline & & 4 & 3 & 968 & 808.6 & 16.5 & 826.9 & & 764 & 36000 & 841.5 & & $1.3 M$ & 36000 & 833.7 & & $88 K$ & 36000 \\
\hline & & & & & & & & Cor & pact & & & Benc & s auto & & Benc & ders & Callbs & acks \\
\hline & & $\mathrm{H}$ & K & BKS & root & root & final & final & nodes & Time & final & final & node: & Time & final & final & nodes & Time \\
\hline & & & & & LB & $\operatorname{gap}(\%)$ & LB & UB & & (s) & LB & UB & & $(\mathrm{s})$ & LB & UB & & (s) \\
\hline & TC-20 & 3 & 2 & 607 & 581.0 & 4.3 & 607.0 & 607 & $2.6 K$ & 43 & 607.0 & 607 & $3.3 K$ & 16 & 607.0 & 607 & $2.8 K$ & 191 \\
\hline & & 3 & 3 & 842 & 798.8 & 5.1 & 842.0 & 842 & $2.9 K$ & 319 & 842.0 & 842 & $11 K$ & 60 & 842.0 & 842 & $11 K$ & 601 \\
\hline & & 4 & 2 & 536 & 501.0 & 6.5 & 536.0 & 536 & $5.2 K$ & 20615 & 536.0 & 536 & $15 K$ & 148 & 536.0 & 536 & $7.1 K$ & 1052 \\
\hline & & 4 & 3 & 750 & 714.8 & 4.7 & 737.2 & 750 & $2.5 K$ & 36000 & 750.0 & 750 & $28 K$ & 392 & 750.0 & 750 & $10 K$ & 1872 \\
\hline & TE-20 & 3 & 2 & 776 & 764.3 & 1.5 & 776.0 & 776 & 47 & 27 & 776.0 & 776 & 13 & 8 & 776.0 & 776 & & 46 \\
\hline & & 3 & 3 & 1082 & 1025.5 & 5.2 & 1082.0 & 1082 & $15 K$ & 8955 & 1082.0 & 1082 & $124 K$ & 1048 & 1082.0 & 1082 & $182 \mathrm{~K}$ & 12865 \\
\hline & & 4 & 2 & 670 & 607.0 & 9.4 & 623.4 & & 834 & 36000 & 670.0 & 670 & $239 K$ & 5454 & 652.3 & & $128 \mathrm{~K}$ & 36000 \\
\hline 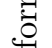 & & 4 & 3 & 919 & 843.2 & 8.3 & 854.4 & & 692 & 36000 & 886.5 & & $881 K$ & 36000 & 876.7 & & $98 K$ & 36000 \\
\hline & TC-40 & 3 & 2 & 632 & 595.8 & 5.7 & 632.0 & 632 & $2.0 K$ & 317 & 632.0 & 632 & $11 K$ & 183 & 632.0 & 632 & 2.4 & 2169 \\
\hline 9 & & 3 & 3 & 889 & 840.1 & 5.5 & 889.0 & 889 & $12 K$ & 11099 & 889.0 & 889 & $141 K$ & 4361 & 882.0 & & $46 K$ & 36000 \\
\hline 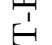 & & 4 & 2 & 538 & 515.8 & 4.1 & 527.5 & & 189 & 36000 & 538.0 & 538 & 868 & 258 & 538.0 & 538 & 817 & 10349 \\
\hline & & 4 & 3 & 795 & 738.6 & 7.1 & 743.1 & & 75 & 36000 & 780.1 & 785 & $297 K$ & 36000 & 763.6 & 785 & $10 K$ & 36000 \\
\hline & TE-40 & 3 & 2 & 790 & 758.3 & 4.0 & 790.0 & 790 & 789 & 688 & 790.0 & 790 & $5.7 \mathrm{~K}$ & 202 & 790.0 & 790 & $2.1 K$ & 2239 \\
\hline & & 3 & 3 & 1094 & 1025.2 & 6.3 & 1062.5 & & $4.18 K$ & 36000 & 1094.0 & 1094 & $456 K$ & 21341 & 1065.3 & & $31 K$ & 36000 \\
\hline & & 4 & 2 & 661 & 614.4 & 7.1 & 618.2 & & 25 & 36000 & 654.0 & $\underline{654}$ & $33 K$ & 5585 & 623.6 & & $1.1 \mathrm{~K}$ & 36000 \\
\hline & & 4 & 3 & 968 & 857.4 & 11.4 & 859.9 & & 23 & 36000 & 883.7 & & $126 K$ & 36000 & 860.5 & & 57 & 36000 \\
\hline
\end{tabular}

Comparison of HOP and DT-HOP formulations: direct solution by CPLEX MIP solver through compact or Benders approaches, and add-hoc branch-and-cut based Benders decomposition. 
Those experiments were carried out on a computer equipped with a processor Intel(R) Xeon(R) CPU X5460 at 3.16GHz and $32 \mathrm{~GB}$ of RAM memory, using Concert Technology for JAVA of CPLEX 12.8 [6]. The time limit was set to 36000 seconds. The TC and TE instances used in tests are widely used the literature. They correspond to complete graphs, vertices are associated to points in the plane, the costs are the Euclidean distances. TC-20 has vertex 0 in the center and demands $D=\{(0, v): v=$ $1, \ldots, 20\}$, TE-20 is similar but has vertex 0 in a corner, TC-40 and TE-40 are similar but $|D|=40$. We performed tests taking $H \in\{3,4\}$ and $K \in\{2,3\}$.

Table 5 is divided into two parts. On the top, it shows results for HOP fomulation; on the bottom for DT-HOP formulation. Columns root $L B$ and root gap show the lower bounds and gap with respect to the Best Known Solution for HOP and DT-HOP formulations (those values do not depend whether Benders decomposition is used or not). The remaining columns are statistics for the exact methods. In particular, the number of nodes are written in thousands $(K)$ or millions $(M)$. Therefore, we are comparing six methods on each instance. We mark in bold the time of the method that could solve the instance faster. If no method could solve the instance within the time limit we mark in bold the best final lower bounds obtained. It can be seen that:

- DT works very well for that problem. For all instances, a method based on DT-HOP was the winner.

- Benders decomposition can be a good alternative for mitigating the problems related to the large size of the reformulations obtained by DT. DT-HOP with automatic Benders was the best method for all instances, allowing us to close most open instances. In addition, the automatic Benders decomposition performs significantly better than the ad-hoc algorithm.

6. Conclusions. A growing part of the Integer Programming research is devoted to finding new effective extended formulations for certain families of problems. This paper contributes in this direction, introducing a technique with the potential of strengthening existing formulations for a large class of NDPs. The increase in formulation size is not necessarily exaggerated (actually, the increase can be controlled by the parameter $L$ ), making the overall approach computationally appealing in a number of cases.

In principle, DT is a very generic technique that could be applied on many other network design problems. The question that has to be answered for any candidate problem is: the reduction of root gaps is significant enough to compensate for the increased size of the formulation? Based on the presented experiments, there are two factors that seem to make the DT more or less suited to a particular NDP.

- The DT seems to work better on NDPs with sparser solutions. For example, on HSND instances with $K=2$ the average gap was reduced by $72 \%$, on HSND instances with $K=3$ the more modest average gap reduction of $43 \%$ was obtained. This is coherent with the theory presented in Section 2 that asserts that DT works by splitting nodes according to the different distances (to the sources) induced by decomposing fractional solutions. Denser fractional solutions provide more ways of performing the decomposition, resulting in less node splittings.

- The DT seems to work better on problems where the solutions are likely to have a "small diameter". For example, this happens on HSND because the of the hop constraint. This allows using small values of $L$ without compromising the strength of the reformulation. Remark that SFP solutions are very sparse, but typically have large diameter. Although the last characteristic is not 
favorable, the DT with limited values of $L$ still provided good results.

There are also important issues that need to be addressed in future research on DT. One of them is devising a general method for choosing the set $S$ and the limit $L$. The experiments on STF with ad-hoc methods showed that those choices can make a lot a difference.

\section{REFERENCES}

[1] YASH P ANEJA, An integer linear programming approach to the Steiner problem in graphs, Networks, 10 (1980), pp. 167-178.

[2] F. Bendali, I. Diarrassouba, A.R. Mahjoub, and J. Mailfert, The $k$ edge-disjoint 3-hopconstrained paths polytope, Discrete Optimization, 7 (2010), pp. 222-233.

[3] Q. Botton, B. Fortz, L. Gouveia, And M. Poss, Benders decomposition for the hopconstrained survivable network design problem, INFORMS Journal on Computing, 25 (2013), pp. 13-26.

[4] Sunil Chopra and Mendu Rammohan Rao, The Steiner tree problem i: Formulations, compositions and extension of facets, Mathematical Programming, 64 (1994), pp. 209-229.

[5] - The Steiner tree problem ii: Properties and classes of facets, Mathematical Programming, 64 (1994), pp. 231-246.

[6] IBM ILOG CPLEX, V12. 1: Users manual for cplex, International Business Machines Corporation, 46 (2009), p. 157.

[7] Michel X. Goemans and David P. Williamson, The primal-dual method for approximation algorithms and its application to network design problems, PWS, Boston, 1997, pp. 144191.

[8] Luis Gouveia, Multicommodity flow models for spanning trees with hop constraints, European Journal of Operational Research, 95 (1996), pp. 178 - 190.

[9] Luis Gouveia, Markus Leitner, and Ivana LuUbić, Hop constrained Steiner trees with multiple root nodes, European Journal of Operational Research, 236 (2014), pp. 100-112.

[10] - The two-level diameter constrained spanning tree problem, Mathematical Programming, 150 (2015), pp. 49-78.

[11] L. Gouveia, L. Simonetti, And E. UchoA, Modeling hop-constrained and diameterconstrained minimum spanning tree problems as Steiner tree problems over layered graphs, Mathematical Programming, 128 (2011), pp. 123-148.

[12] D. Huygens And A.R. Mahjoub, Integer programming formulations for the two 4-hopconstrained paths problem, Networks, 49 (2007), pp. 135-144.

[13] D. Huygens, A.R. Mahjoub, and P. Pesneau, Two edge-disjoint hop-constrained paths and polyhedra, SIAM Journal on Discrete Mathematics, 18 (2004), pp. 287-312.

[14] Thorsten Koch, Alexander Martin, and Stefan Voss, Steinlib: An updated library on Steiner tree problems in graphs, in Steiner trees in industry, Springer, 2001, pp. 285-325.

[15] Jochen Könemann, Stefano Leonardi, Guido Schäfer, and Stefan HM van Zwam, $A$ group-strategyproof cost sharing mechanism for the Steiner forest game, SIAM Journal on Computing, 37 (2008), pp. 1319-1341.

[16] Ivana Lunbic and Stefan Gollowitzer, Layered graph approaches to the hop constrained connected facility location problem, INFORMS Journal on Computing, 25 (2013), pp. 256270.

[17] Thomas L Magnanti and S Raghavan, Strong formulations for network design problems with connectivity requirements, Networks, 45 (2005), pp. 61-79.

[18] A. R. Mahjoub, L. Simonetti, And E. UchoA, Hop-level flow formulation for the survivable network design with hop constraints problem, Networks, 61 (2013), pp. 171-179.

[19] M. Poggi de Aragão, E. Uchoa, and R.F. Werneck, Dual heuristics on the exact solution of large Steiner problems, Electronic Notes in Disc. Mathematics, 7 (2001), pp. 150-153.

[20] T. Polzin And S.V. Daneshmand, Improved algorithms for the Steiner problem in networks, Discrete Applied Mathematics, 112 (2001), pp. 263-300.

[21] Mario Ruthmair and Günther R RAidL, A layered graph model and an adaptive layers framework to solve delay-constrained minimum tree problems, in International Conference on Integer Programming and Combinatorial Optimization, Springer, 2011, pp. 376-388.

[22] D. Schmidt, B. Zey, and F. Margot, MIP Formulations for the Steiner Forest Problem, ArXiv e-prints, (2017).

[23] R.T. Wong, A dual ascent approach for Steiner tree problems on a directed graph, Mathematical programming, 28 (1984), pp. 271-287. 